\title{
A Fuzzy-Stochastic Multiscale Model for Fiber Composites
}

\section{A one-dimensional study}

\author{
Ivo Babuška ${ }^{\mathrm{a}}$, Mohammad Motamed ${ }^{\mathrm{b}, *}$ \\ ${ }^{a}$ Institute for Computational Engineering and Sciences, The University of Texas at Austin, USA \\ ${ }^{b}$ Department of Mathematics and Statistics, The University of New Mexico, Albuquerque, USA
}

\begin{abstract}
We study mathematical and computational models for computing the deformation of fiberreinforced polymers due to external forces. A thorough study requires an understanding of both micro-structural effects and uncertainty/variability in the manufacturing process, such as uncertainty in the size and distribution of fibers and variability in material properties and fracture parameters. We first show that the present uncertainties/variabilities, which are of both random and non-random types, cannot be accurately characterized by current stochastic multiscale models based on precise probability theory, such as stationary random fields. Next, we present a new hybrid fuzzy-stochastic model, which can more accurately describe uncertainties/variabilities in fiber composites. The new model, which is referred to as a fuzzy-stationary random field, consists of a random field with fuzzy moments. We then construct a global-local multiscale algorithm in a fuzzy-stochastic framework for efficiently computing output quantities of interest, such as displacements and stresses, in regions of relatively small size, e.g. hot spots. The algorithm utilizes the concept of representative volume elements and homogenization and constructs a global solution to compute a local approximation that captures the microscale features of the problem. The results are based on and backed by real experimental data through a calibration-validation approach.
\end{abstract}

Keywords: fiber composites, uncertainty quantification, precise probability, imprecise uncertainty, stochastic models, fuzzy variables, multiscale simulation, global-local method

\section{Introduction}

Fiber-reinforced composite materials are widely used in aerospace, marine, and automotive industries. They consist of stiff fibers in a matrix which is less stiff. In composites with unidirectional fibers, a large number of long unidirectional fibers are aligned in a thin ply. To achieve high stiffness, a few plies are stacked together, each having fibers oriented in a certain direction. Such a stack is termed a cross-plied laminate; see Figure 1.

Fiber composites are fabricated based on their response to external forces. Two principal responses are deformation and fracture. In a non-testing environment, we need accurate

\footnotetext{
*Corresponding author

Email addresses: babuska@ices.utexas.edu (Ivo Babuška), motamed@math.unm.edu (Mohammad Motamed)
} 


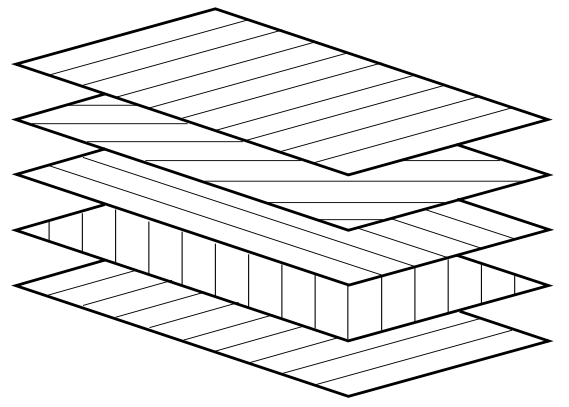

(a) Plies with fibers in different directions

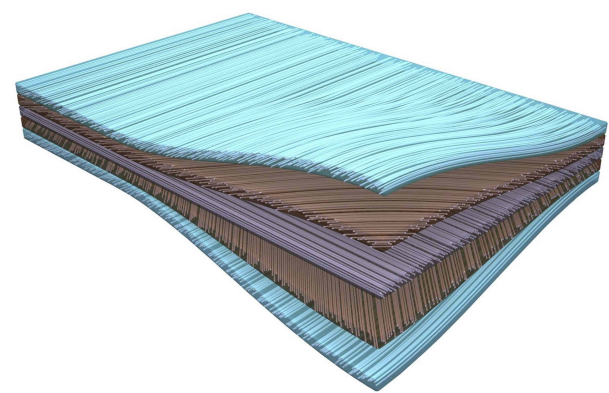

(b) Plies are stacked to form a laminate

Figure 1: A cross-plied fiber composite laminate made by stacking five plies. Different plies contain many unidirectional fibers in different directions aligned in a matrix.

and viable mathematical and computational models for computing stress distributions and predicting damage and failure. This requires an understanding of both micro-structural effects and uncertainty/variability in the manufacturing process. High fidelity models must therefore account for two components: multiple scales and uncertainty. On the one hand, the problem involves multiple length scales ranging from the diameter of fibers $(\sim 10$ micron $)$ to the laminate thickness and length $(\sim 1$ meter $)$. On the other hand, the model is subject to uncertainty, due to the random character of the size and distribution of fibers and the intrinsic variability in material properties and fracture parameters. High levels of confidence in the predictions require a process called multiscale uncertainty quantification (UQ) taking into account the interaction between the two involved components, i.e. multiple scales and uncertainty.

A chronological development of mathematical and computational tools for solving multiscale problems (such as fiber composites) may in general be classified into four groups:

1. Mathematical theory of homogenization: The term "homogenization" was first coined by Babuška $[4,6]$. Homogenization is an analytical approach to replace the multiscale problem with heterogeneous coefficients by an equivalent problem with homogeneous coefficients, known as the homogenized problem. Homogenization theory is well studied in the case of periodic or locally periodic microscale coefficients [17]. For the more general case of non-periodic coefficients, homogenization theory is carried out by studying the G-convergence [72] and H-convergence [63] of solution operates.

2. Multiscale numerical methods: Numerical methods that approximate the highly oscillatory solution of a multiscale problem by solving an effective problem and including local microscale oscillations instead of directly solving the original problem are called multiscale numerical methods. Numerical homogenizetion $[5,7,8,9]$ is the earliest multiscale method, where the finite element method was employed to solve the homogenized problem and some microscale features were added as correctors to the homogenized solution. Since then, many multiscale methods have been proposed, including the generalized finite element method [15, 60], the variational multiscale method [48], the multiscale finite element method [29], projection-based numerical homogenization $[23,32]$, the heterogeneous multiscale method [27, 28], and the equation-free method 
[53]. We also refer to $[47,26,33]$ for detailed discussions on a wide range of multiscale methods. All these techniques usually seek the approximate solution everywhere inside the computational domain. Another class of multiscale methods is based on a globallocal approach [64, 74, 11], where the solution or other quantities of interest (QoIs) are required inside a relatively small subdomain. In these methods, a global (homogenized) solution is employed to recover the microscale solution inside the local subdomain.

3. Multiscale methods in engineering: There is a vast literature on multiscale methods in engineering, especially in the field of materials science. These methods have been developed based on the same ideas and principles employed in the applied mathematics community. They have been proposed to account for microstructural heterogeneity, for instance in complex materials such as composites and porous structures. They include the unit cell or representative volume element (RVE) method, multi-level approaches using the finite element method and the Voronoi cell finite element method, and continuousdiscontinuous homogenization; see for instance [35, 56, 34, 71, 55, 54, 52, 38, 39, 73]. These multiscale methods treat both linear and non-linear mechanical and thermomechanical responses of complex materials.

4. Probabilistic treatment of multiscale problems: The literature on UQ for multiscale problems is rather sparse and focuses more on stochastic models. Stochastic homogenization $[70,19,18,41]$ can be considered as a generalization of classical homogenization. Theoretical aspects of stochastic homogenization is well studied in the case of stationary and ergodic random fields. However, numerical approaches based on stochastic homogenization are cumbersome and not well studied, particularly because the homogenized problem is set on the whole space, not on a finite cell. Analogous to the case of deterministic problems, a variety of stochastic multiscale methods have been proposed within the framework of variational multiscale methods, multiscale finite element methods, the heterogeneous multiscale methods, and the global-local approach; see e.g. $[2,37,1,59,20,12]$. Also in engineering community, an increasing number of papers are attempting to address UQ in multiscale simulations; see e.g. [76, 44, 57, 58, 65].

In the particular case of composite materials, a majority of multiscale models are deterministic; see references in item 3 above. Such models are not capable of fully describing the mechanical behavior of composites, partially due to the ignorance of uncertainty which is an important component that must be included in the model. More recently, there have been efforts to include and describe uncertainty by precise probabilities [43], where the model input parameters, i.e. material properties such as the modulus of elasticity, are described by (often stationary Gaussian or log-normal) random fields; see references in item 4 above. Despite recent advances with stochastic multiscale models and UQ methodologies, five decades after the pioneering work of Kachanov [49,50] on continuum damage mechanics, one question still remains open: are there viable models for the sound investigation of composite responses? As Rohwer [67] has recently remarked, "A fully satisfying model for describing damage and failure of fiber composites is not yet available. Consequently, for the time being, a real test remains the authentic way to secure structural strength." Beside the deficiency of accurate models for damage mechanisms, one reason for the inapplicability of current stochastic 
multiscale models is the presence of both random (statistical or aleatoric) and non-random (systematic or epistemic) uncertainties in the problem and the scarcity of noisy measurements that are used to characterize uncertainties. In order to describe both types of uncertainties, we need to develop more sophisticated uncertainty models beyond precise probabilities.

In the present paper, we are concerned with mathematical and computational models for computing the deformation of fiber composites due to external forces. Since realistic models must be designed based on and backed by real experimental data, we consider a small piece of a real fiber composite plate, taken from [10], consisting of four plies and containing 13688 unidirectional fibers with a volume fraction of $63 \%$. The measured raw data include the material constants of fibers and the matrix, and a map of the size and distribution of fibers, obtained by an optical microscope. The map is considered as a prototype of fiber distributions in fiber composites. We process the raw data and convert them into a form suitable for statistical analysis and show that the current stochastic models are not capable of correctly characterizing uncertainty in fiber composites. Instead, we study and show the applicability of imprecise uncertainty models for fiber composites. We propose a new hybrid fuzzy-stochastic model by integrating probability theory [43] and fuzzy set theory [77] through a calibration-validation approach. We finally present a numerical method in a fuzzystochastic framework for propagating uncertainty through the proposed model and predicting output QoIs. The numerical method utilizes the concept of RVEs and homogenization and is based on a global-local approach, where a global solution is used to construct a local solution that captures the microscale features of the problem. For simplification and to motivate and establish the main concepts of the proposed model, we consider a one-dimensional problem. The present work is a preparation for studying fiber composites in two and three dimensions, which will be presented elsewhere.

The main contributions of this paper include: (1) showing the deficiency of stochastic models to the reliable prediction of fiber composite responses; (2) motivating the applicability of imprecise uncertainty models and constructing a novel hybrid fuzzy-stochastic model for the uncertainty characterization of fiber composites; and (3) developing a global-local numerical method in a fuzzy-stochastic framework for efficiently computing the output QoIs.

The rest of the paper is organized as follows. In Section 2, we present the real data, formulate the problem, and briefly address different models for characterizing uncertainty. In Section 3, we perform statistical analysis and motivate the deficiency of current stochastic models. We outline the basic concepts of fuzzy set theory and its combination with probability theory in Section 4. In Section 5, we discuss the construction of the new hybrid fuzzystochastic model. In Section 6, we present the numerical multiscale method. Finally, we summarize our conclusions and outline future works in Section 7.

\section{Problem Statement}

Reliable mathematical and computational models for predicting the response of fiber composites due to external forces must be designed based on and backed by real experimental data. In this section, we first present the real data that is used throughout this work. We then formulate a simple one-dimensional problem describing the deformation of fiber composites. Finally, we briefly address different models for characterizing uncertainty in the problem. 


\subsection{Real data}

The real data that we use are obtained from a small piece of a HTA/6376 carbon fiberreinforced epoxy composite plate $[10,12]$ with a rectangular cross section of size $1.7 \times 0.5 \mathrm{~mm}^{2}$, and consisting of four plies containing 13688 unidirectional fibers with a volume fraction of $63 \%$. Fiber diameters vary between $4 \mu \mathrm{m}$ to $10 \mu \mathrm{m}$. Figure 2 shows a map of the size and position of fibers in an orthogonal cross section of the composite obtained by an optical microscope. In the present work, this particular map serves as a prototype of fiber distributions in fiber composites.
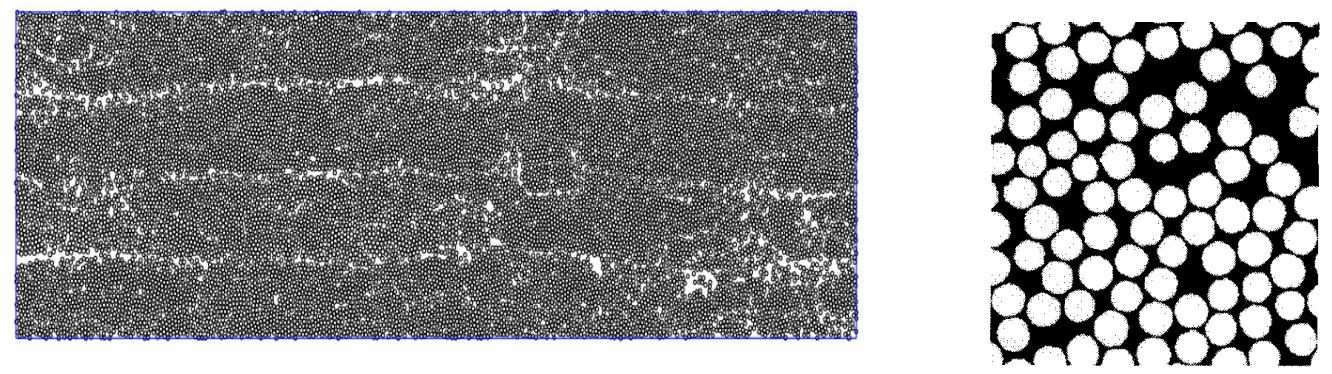

Figure 2: Left: A $1.7 \times 0.5 \mathrm{~mm}^{2}$ rectangular orthogonal cross section of a small piece of a fiber composite laminate consisting of four uni-directional plies containing 13688 fibers with a volume fraction of $63 \%$. Right: A binary image of a small part of the whole micrograph.

The Young's modulus of elasticity and Poisson's ratio of the fiber composite under consideration are given in Table 1.

Table 1: Material constants for the composite under consideration.

\begin{tabular}{ccc}
\hline composite phases & $a$ & $\nu$ \\
\hline fiber & $24[\mathrm{GPa}]$ & 0.24 \\
matrix & $3.6[\mathrm{GPa}]$ & 0.3 \\
\hline
\end{tabular}

\subsection{Mathematical formulation: a one-dimensional problem}

The deformation of elastic materials is given by the elastic partial differential equations (PDEs) in three dimensions. In the particular case of plane strain, where the length of structures in one direction is very large compared to the size of structures in the other two directions, the problem may approximately be reduced to a two-dimensional problem. In the present work, however, for simplification and to motivate and establish the main concepts of the proposed model, we consider a one-dimensional problem. Problems in higher dimensions will be presented elsewhere.

We consider the elastic equation with homogeneous Dirichlet and non-homogeneous Neumann boundary conditions in one dimension:

$$
\begin{gathered}
\frac{d}{d x}\left(a(x) \frac{d u}{d x}(x)\right)=f(x), \quad x \in[0,1], \\
u(0)=0, \quad a(1) \frac{d u}{d x}(1)=1,
\end{gathered}
$$


where $x$ is location, $u(x)$ is the displacement, $a(x)$ is the modulus of elasticity of the composite, and $f(x)$ is a force term, given for instance by,

$$
f(x)= \begin{cases}2 & x \in[0,0.5) \\ 0 & x \in[0.5,1]\end{cases}
$$

Here, the unit of length is assumed to be meter [m], and the unit of force (both external force $f$ and the boundary force) and modulus of elasticity $a$ is assumed to be giga Pascal [GPa].

The main goal of computations is to obtain composite deformations due to external forces. We therefore need to solve problem (1) and compute displacements $u(x)$, stresses $a(x) u^{\prime}(x)$, and/or other QoIs (for example functionals of the displacement $u(x)$ ). We note that in the one-dimensional model problem considered here, stresses are smooth functions and do not oscillate with the small scale of fiber sizes. We therefore set our main goal as the prediction of the solution at a given point, say at $x_{0}=0.75$. We then introduce the QoI:

$$
\mathcal{Q}=u\left(x_{0}\right), \quad x_{0}=0.75 .
$$

Here, the solution to the problem (1) is analytically given by

$$
u(x)=\int_{0}^{x} a^{-1}(\xi) F(\xi) d \xi, \quad F(\xi)=\int_{0}^{\xi} f(\tau) d \tau= \begin{cases}2 \xi & \xi \in[0,0.5), \\ 1 & \xi \in[0.5,1] .\end{cases}
$$

To obtain the solution and the QoI in (2), we need to know the parameter $a(x)$, which describes the mechanical property of the composite. Therefore, we first need to characterize the modulus of elasticity $a(x)$, which is directly given by the size and position of fibers in the matrix and by the modulus of elasticity of fibers and matrix, listed in Table 1.

The parameter $a(x)$ needs to be modeled based on real data provided by the manufacturer. Moreover, as mentioned in introduction, two major difficulties arise in modeling the material parameter. First, the parameter is highly oscillatory, because there are a large number of small fibers distributed in the matrix. A direct representation is therefore not practical due to computational power limitations and unavailability of data for the whole structure. Secondly, uncertainty - due to various sources - must be included in the expression of the parameter.

\subsection{On uncertainty characterization and stochastic models}

Current models for characterizing the parameter $a(x)$ are based on stochastic representation of uncertainty. In practice, the parameter is assumed to be a stationary (and often Gaussian or lognormal) random field with known marginal probability distributions. However, as we will show later, stochastic models in general, and stationary fields in particular, are not able to correctly represent the uncertainty in material properties. Other models need to be considered. In general, the uncertainty in $a$ can be classified into two categories:

I. Precise or deterministic uncertainty. This uncertainty is associated with an identifiable but unknown or uncertain source. In this case, we exactly know the uncertainty in the parameter. Two common models include: (1) stochastic models, where $a$ is modeled by random variables or random fields with known and crisp distributions and covariances; and (2) fuzzy models, where $a$ is modeled by fuzzy variables or fuzzy fields with known and crisp membership functions; see Section 4 for the definition of membership function. 
II. Imprecise or non-deterministic uncertainty. In this case, we do not exactly know the uncertainties in the parameter. The uncertainty characterization can be carried out by different combinations of the above two models. A few examples include: (1) mixed stochastic models, where $a$ is modeled by a random field, whose mean and variance are random variables, (2) mixed fuzzy models, where $a$ is modeled by a fuzzy field, whose mean is a fuzzy number; and (3) fuzzy-stochastic models, where $a$ is modeled by a random field, whose mean and variance are fuzzy numbers.

Precise uncertainty, and particularly stochastic models, can be used to characterize uncertainty in physical systems in the presence of abundant, accurate data. In many real applications such as fiber composites, however, the available data are scarce and imprecise. In such cases, the parameters cannot be accurately characterized by precise uncertainty models. Moreover, as we will show in details in the next section, the material properties are not stationary fields. The non-stationarity is also visible from the map of fibers in Figure 2. In the rest of the paper, we first show that stochastic models may not be capable of correctly characterizing uncertainty in fiber composites. We then propose a combined fuzzy-stochastic model for the uncertain microstructure parameter $a$.

\section{Statistical analysis}

In this section we perform statistical analysis on the data. We first describe data collection procedures. We then study statistical moments and correlation length of the parameter field.

\subsection{Data collection}

A binary data map. The available data contains the center's location and area of all 13688 fibers in the orthogonal cross section $D \subset \mathbb{R}^{2}$ of the composite shown in Figure 2. We first discretize the two-dimensional domain $D$ into a uniform mesh consisting of $500 \times 1700$ square elements (pixels) of size $1 \times 1 \mu \mathrm{m}^{2}$. We then construct a binary data structure, where the presence or absence of fiber at every pixel is marked by 1 or 0 , respectively, assuming that fibers are perfectly circular. Using the binary structure generated above, the material elasticity moduli are recovered. For instance, to compute Young's modulus, at each pixel with labels 1 or 0 , we put $a=a_{\text {fiber }}=24$ [Gpa] or $a=a_{\text {matrix }}=3.6$ [Gpa], respectively. We note that significant experiments have been performed for validating the binary mapping procedure and the size of square elements or pixels. It was found that a size of $1 \mu \mathrm{m}$ provides the desired accuracy without being too computationally expensive. In particular, the binary map with elements of size 1 micron is accurate within $1 \%$ in predicting the overall volume fraction obtained by an analytic (and expensive) approach.

One-dimensional data collection. To obtain one-dimensional data samples from the binary data map, we proceed as follows. First, we divide the rectangular cross section of the composite $D$ into $M=50$ thin horizontal strips (or bars) of width $h=10 \mu \mathrm{m}$ and length $1700 \mu \mathrm{m}$ along the $x$-axis. This gives us $M$ thin bars labeled $m=1, \ldots, M$. By the binary-micron map constructed above, the modulus of elasticity in each bar is given on a mesh of $10 \times 1700$ one-micron pixels. Next, each bar $m$ is divided into 170 square elements of size $10 \mu \mathrm{m}$, labeled $j=1, \ldots, 170$; see Figure 3 (top). Each element $j$ in a bar contains 
$10 \times 10$ pixels. On each element $j$, we take the harmonic average over its $10 \times 10$ pixels and compute a one-dimensional modulus of elasticity $a_{m}\left(x_{j}\right)$. We repeat the process for all 170 elements along $x$ axis and compute $\left\{a_{m}\left(x_{j}\right)\right\}_{j=1}^{170}$. This is considered as a sample of the uncertain parameter $a(x)$; see Figure 3 (bottom). Eventually we compute and collect all $M=50$ samples by repeating the same steps for all thin bars and obtain $M$ one-dimensional samples $\left\{a_{m}\left(x_{j}\right)\right\}_{m=1}^{M}$ at the discrete points $\left\{x_{j}\right\}_{j=1}^{170}$.

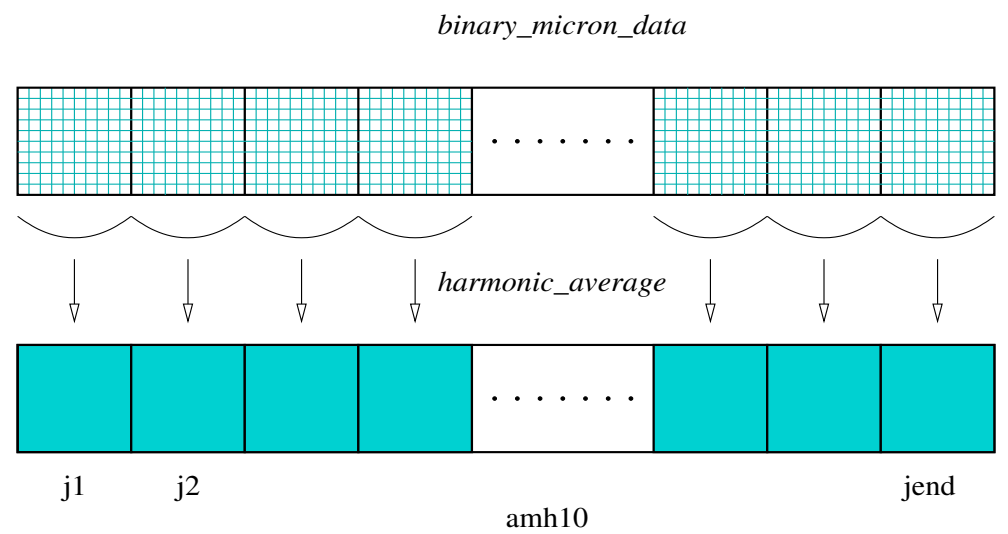

Figure 3: A schematic representation of deriving 1D data samples (bottom) from the binary data given on the $1 \times 1 \mu \mathrm{m}$ pixels (top). On each element $j$, we take the harmonic average of $a$ on its $10 \times 10$ pixels.

We note that the parameter $a(x)$ involves multiple scales and is highly oscillatory. The small variations in $a(x)$ are of the size of fibers $(\sim 10 \mu \mathrm{m})$ and are much smaller (10 $0^{5}$ times) than the length of the whole domain $(\sim 1 \mathrm{~m})$. We obtain the samples on elements of size $10 \mu \mathrm{m}$ in order to fully resolve the micro scales. The harmonic averaging is also motivated by one-dimensional periodic homogenization. We emphasize however that since the size of elements are approximately of the size of fibers, the samples obtained here are not homogenized, and they correspond to a full resolution of the micro scale. We will use homogenization in Section 6, where a global-local approach will be presented to solve the multiscale problem.

Bootstrapping. To this end, we have $M=50$ discrete and highly oscillatory samples of $a(x)$ given on $x \in[0,1.7] \mathrm{mm}$. Each sample corresponds to a one-dimensional bar of length $L=1.7$ $\mathrm{mm}$. In practice, we may need more samples $(M>50)$ of bars with longer lengths $(L>1.7$ $\mathrm{mm})$, particularly because the length of the composite plate $(1 \mathrm{~m})$ is much larger than the length of the available data $(1.7 \mathrm{~mm})$. This can be done by bootstrapping technique [31, 30]. For example, suppose that from the original data set (i.e. 50 samples of length $1.7 \mathrm{~mm}$ ), we want to generate $M=100$ bootstrap samples of length $L=10 \mathrm{~mm}$. A bootstrap sample is generated by randomly sampling $n=\lceil 10 / 1.7\rceil=6$ times, with replacement, from the original set and putting them next to each other. This generates a bar of length $6 * 1.7=10.2 \mathrm{~mm}$. The extra $0.2 \mathrm{~mm}$ is then removed to obtain a sample of length $10 \mathrm{~mm}$. We repeat this process and obtain the rest of $M-1$ bootstrap samples of length $10 \mathrm{~mm}$. This procedure can be performed for any given number of samples $M$ and any desired length $L$. The new $M$ bootstrap samples of length $L$, denoted by $\left\{a_{m}\left(x_{j}\right)\right\}_{m=1}^{M}$ at $N_{x}=1+L[\mu \mathrm{m}] / 10$ discrete points $\left\{x_{j}\right\}_{j=1}^{N_{x}} \in[0, L]$ will be used as the data for statistical analysis. 


\subsection{First four statistical moments}

Motivated by the analytical form of the solution (3), we will perform statistical analysis on the reciprocal of $a$ instead of $a$ itself. We therefore set $b(x)=a^{-1}(x)$ and carry out the analysis using $M$ discrete bootstrap samples $\left\{b_{m}\left(x_{j}\right)\right\}_{m=1}^{M}$ of length $L$, obtained in Section 3.1 , where $b_{m}\left(x_{j}\right)=a_{m}^{-1}\left(x_{j}\right)$. As an example in this section, we choose $M=100$ and $L=10$ $\mathrm{mm}$, and approximate the first four moments of the field $b(x)$ at each discrete point $\left\{x_{j}\right\}_{j=1}^{N_{x}}$ using $M=100$ samples $\left\{b_{m}\left(x_{j}\right)\right\}_{m=1}^{M}$ of length $L=10 \mathrm{~mm}$ :

$$
\begin{gathered}
\mu\left(x_{j}\right)=\frac{1}{M} \sum_{m=1}^{M} b_{m}\left(x_{j}\right), \quad \sigma^{2}\left(x_{j}\right)=\frac{1}{M} \sum_{m=1}^{M}\left(b_{m}\left(x_{j}\right)-\mu\left(x_{j}\right)\right)^{2}, \\
\gamma_{1}\left(x_{j}\right)=\frac{1}{M} \sum_{m=1}^{M}\left(\frac{b_{m}\left(x_{j}\right)-\mu\left(x_{j}\right)}{\sigma}\right)^{3},
\end{gathered}
$$

Here, $\mu, \sigma, \gamma_{1}$, and $\gamma_{2}$ are sample mean, sample standard deviation, sample skewness, and sample excess kurtosis, respectively. Figure 4 shows the sample first four moments of the parameter $b(x)$ versus $x$, and their histograms. It is important to note that the moments of the 100 bootstrap samples, shown in Figure 4, are qualitatively the same as the moments of the 50 original samples. In particular, the histograms of the moments of bootstrap data are almost identical with the histograms of the moments of original data. This important observation justifies the bootstrap procedure used in our data processing and indicates that the results obtained by the bootstrap data set do not deviate from the results obtained by the original data set.

Obviously, the moments are not constant and vary rapidly in $x$. This shows that the uncertain parameter $b(x)$ cannot be accurately represented by stationary random fields. It also shows that the field is not Gaussian, since for instance the skewness is not zero. We therefore need to consider other models than stationary Gaussian random fields to have a better characterization of uncertainty in the parameter $a(x)$. One option is to construct a non-stationary and non-Gaussian random field with variable moments. There are however three problems with this option. One main issue is that due to the imprecise character of uncertainties in the problem, it is not possible to accurately describe the moments by crisp values. Secondly, it is often difficult in practice to work with general non-stationary random fields. For instance, a Karhunen-Loeve approximation of such general fields may require iterative solvers based on density estimation approaches, see e.g. [66, 75]. Moreover, from a computational point of view, numerical stochastic homogenization techniques, which is necessary to treat the multiscale nature of the parameter, are not applicable in the case of non-stationary fields [70, 19, 18, 41].

In Section 5, we propose an alternative option, where instead of considering and dealing with a non-stationary random field with variable crisp moments, a fuzzy-stationary random field is considered, i.e. a random field whose first four moments are fuzzy variables and independent of $x$. Such models are both easy to construct and allow for including imprecise uncertainty in the problem. 

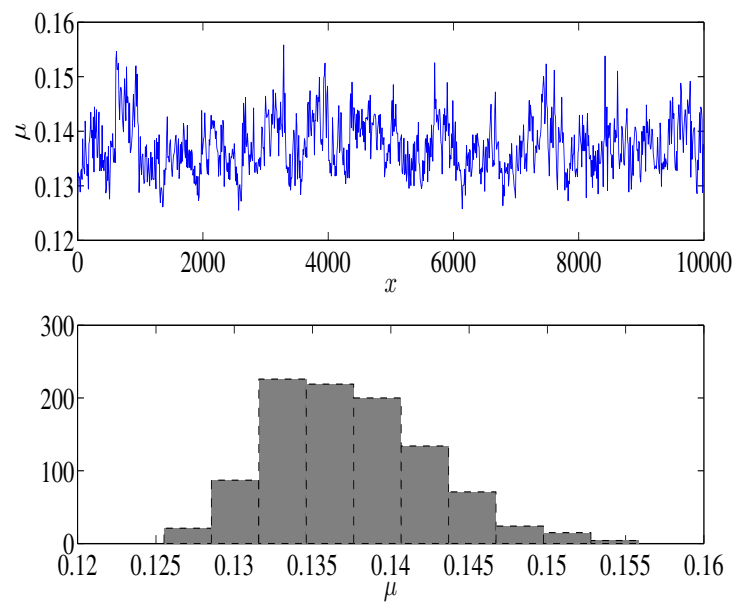

(a) sample mean
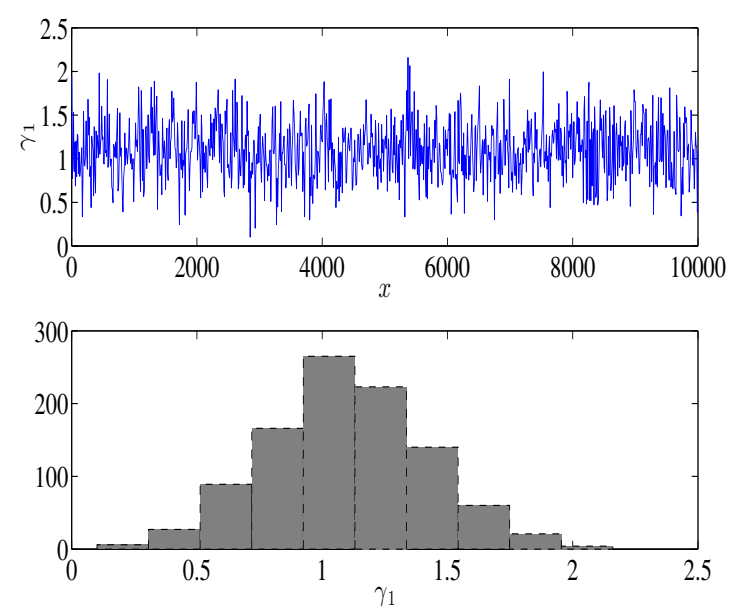

(c) sample skewness
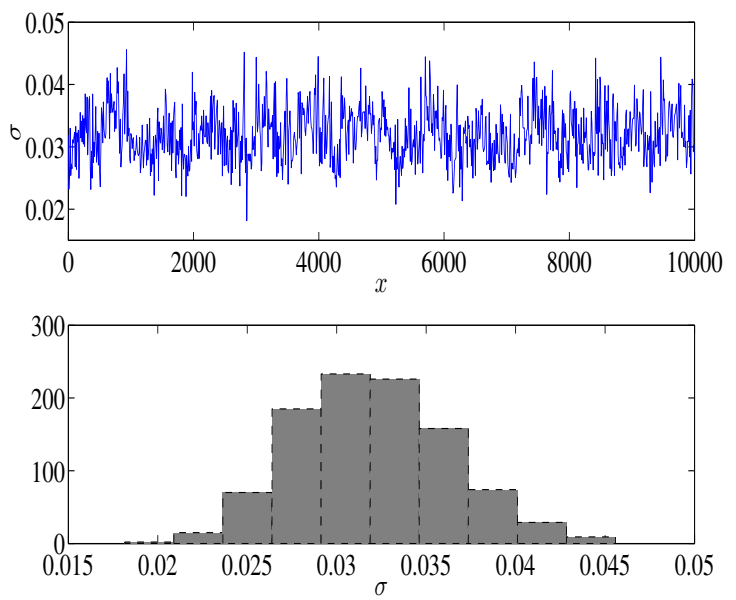

(b) sample standard deviation
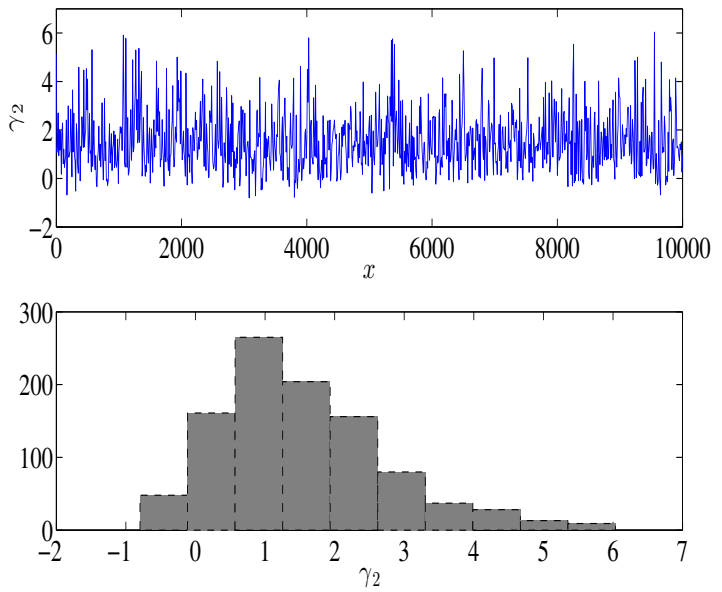

(d) sample excess kurtosis

Figure 4: Sample moments of the field $b(x)=a^{-1}(x)$ versus $x$ and their histograms.

\subsection{Correlation length and correlation function}

We now study the correlation length $\ell$ of the one-dimensional field $b(x)$. Let $b_{m}(x)$ be the $m$-th realization of the field, corresponding to the $m$-th thin bar, given on a set of $N_{x}$ discrete points $\left\{x_{j}\right\}_{j=1}^{N_{x}}$ along the thin bar. The mean of the field can be estimated by the average of the $m$-th sample over all discrete points

$$
\hat{\mu}_{m}=\frac{1}{N_{x}} \sum_{j=1}^{N_{x}} b_{m}\left(x_{j}\right) .
$$

For an increasing sequence of distances $r_{n}=n h$, with $n=1,2,3, \ldots$, and $h=10 \mu \mathrm{m}$, we find all pairs $\left(x_{i}, x_{j}\right)$ separated by distance $r_{n} \pm \frac{h}{2}$ : find $(i, j)$ s.t. $\left|x_{i}-x_{j}\right| \in\left[r_{n}-\frac{h}{2}, r_{n}+\frac{h}{2}\right]$. 
For such pairs, we introduce the normalized empirical correlation function

$$
C_{m}\left(r_{n}\right)=\frac{\sum_{(i, j)}\left(b_{m}\left(x_{i}\right)-\hat{\mu}_{m}\right)\left(b_{m}\left(x_{j}\right)-\hat{\mu}_{m}\right)}{\sqrt{\sum_{i}\left(b_{m}\left(x_{i}\right)-\hat{\mu}_{m}\right)^{2}} \sqrt{\sum_{j}\left(b_{m}\left(x_{j}\right)-\hat{\mu}_{m}\right)^{2}}} .
$$

We repeat the above procedure for all $M$ realizations. Figure 5 shows the normalized correlation function $C_{m}\left(r_{n}\right)$ versus $r_{n}$ for all realizations $m=1, \ldots, M$.

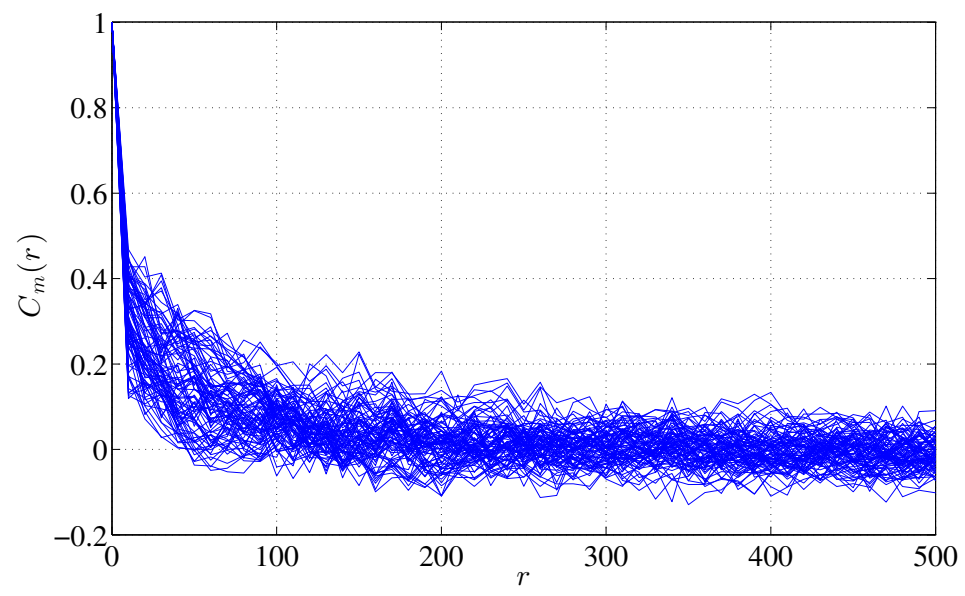

Figure 5: Empirical correlation function $C_{m}\left(r_{n}\right)$ versus the distance $r_{n}$ for the parameter $b_{m}(x)$ for all realizations $m=1, \ldots, M$.

We observe that the correlation function decreases from 1 at $r=0$ by $50 \%$ at $r=10$, by $70 \%$ at a distance close to $r=50$, by $80 \%$ at a distance close to $r=100$, and continues decreasing by more than $90 \%$ at larger distances. This suggests that the correlation length of the parameter field $b(x)$ is of the order of fiber diameters $(\sim 5 h-20 h)$. We will use this observation in Section 5 to construct the empirical field. In practice, the value of correlation length for the empirical field must be selected so that the best fit to the data is obtained.

\section{Fuzzy set theory and fuzzy randomness}

The hybrid fuzzy-stochastic model that will be developed in Section 5 relies on a novel integration of fuzzy set theory and probability theory. Hence, we first provide a brief overview of the basic concepts, such as fuzzy variables, fuzzy functions, and fuzzy random functions, that are relevant to the focus of the present paper. We refer to $[25,22,3,68,16,61]$ for detailed discussions and applications of fuzzy set theory and fuzzy randomness.

\subsection{Fuzzy variables and fuzzy vectors}

Fuzzy sets, introduced by Zadeh [77], generalize the notion of crisp sets. In a crisp set, an element either belongs to or does not belong to the set. The notion of membership in this case can be viewed as a characteristic function, taking values either 0 or 1 to characterize the absence or presence of elements in the set. In a fuzzy set, an element is allowed to be partially in the set. Each element is given a degree of membership, ranging from 0 (not a member) to 1 (a member); see Figure 6. 


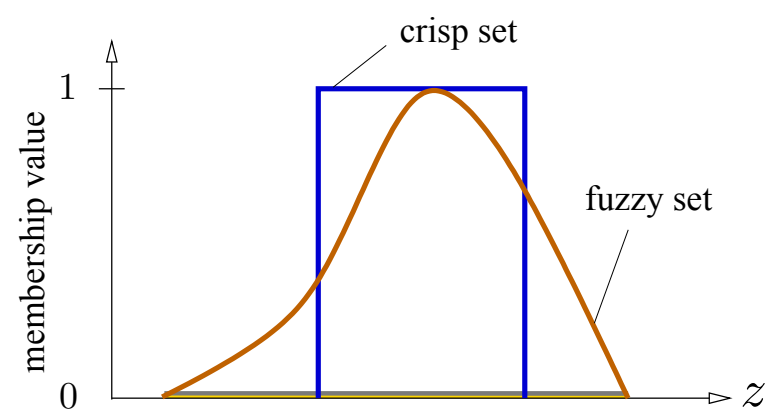

Figure 6: The notion of membership in crisp sets and fuzzy sets.

More precisely, let $Z \subset \mathbb{R}$ be a crisp set of elements, called the universe, whose generic elements are denoted by z. A fuzzy set, or fuzzy variable, is defined by a set of pairs

$$
\tilde{z}=\{(z, \mu(z)) \mid z \in Z, \mu(z): \mathbb{R} \rightarrow[0,1]\},
$$

where $\mu$ is a continuous membership function. We further assume that the fuzzy set is convex:

$$
\forall \lambda \in[0,1] \text { and } \forall z_{1}, z_{2} \in Z: \mu\left(\lambda z_{1}+(1-\lambda) z_{2}\right) \geq \min \left(\mu\left(z_{1}\right), \mu\left(z_{2}\right)\right) \text {. }
$$

We note that the convexity of a fuzzy set does not necessarily imply the convexity of the membership function. In fact, for a fuzzy set to be convex, we need its membership function to be monotonically decreasing on each side of the maximum value.

An important notion in fuzzy set theory is the notion of $\alpha$-cuts, which allows one to decompose fuzzy computations into several interval computations [62]. The membership function $\mu(z)$ can be identified with the one-parametric family of its $\alpha$-level sets, or $\alpha$-cuts, $S(\alpha), \alpha \in[0,1]$, given by

$$
\forall \alpha \in(0,1]: S(\alpha)=\{z \in Z \mid \mu(z) \geq \alpha\}, \quad \text { and } \quad S(0)=\operatorname{closure}\{z \in Z \mid \mu(z)>0\}
$$

An $\alpha$-cut is a closed connected crisp interval with $S\left(\alpha_{2}\right) \subset S\left(\alpha_{1}\right)$ if $\alpha_{2} \geq \alpha_{1}$; see Figure 7 .

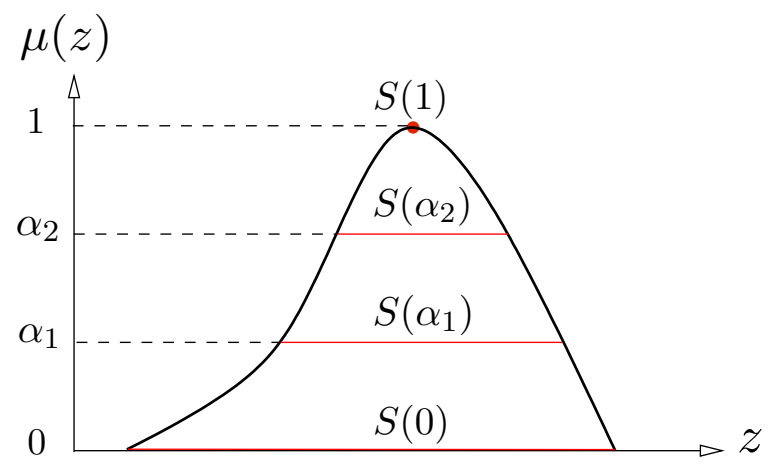

Figure 7: $\alpha$-cuts of a fuzzy variable. 
As the $n$-dimensional generalization of a fuzzy variable, an $n$-dimensional fuzzy vector is defined by a set of pairs

$$
\tilde{\mathbf{z}}=\left\{(\mathbf{z}, \mu(\mathbf{z})) \mid \mathbf{z} \in Z \subset \mathbb{R}^{n}, \mu(\mathbf{z}): \mathbb{R}^{n} \rightarrow[0,1]\right\} .
$$

where $\mu$ is a continuous joint membership function. Analogous to the one-dimensional case, the joint membership function can be identified with the one-parametric family of $n$-dimensional joint $\alpha$-cuts given by

$$
\forall \alpha \in(0,1]: \quad S(\alpha)=\left\{\mathbf{z} \in \mathbb{R}^{n} \mid \mu(\mathbf{z}) \geq \alpha\right\}, \quad \text { and } \quad S(0)=\operatorname{closure}\left\{\mathbf{z} \in \mathbb{R}^{n} \mid \mu(\mathbf{z})>0\right\} .
$$

An important issue in fuzzy arithmetic is the interaction of fuzzy variables, which is analogous to the correlation of random variables. The interaction of fuzzy variables can be expressed using the notion of $\alpha$-cuts. Let $\tilde{z}_{i}=\left\{\left(z_{i}, \mu_{i}\left(z_{i}\right)\right) \mid z_{i} \in Z_{i} \subset \mathbb{R}, \mu_{i}\left(z_{i}\right): \mathbb{R} \rightarrow[0,1]\right\}$, with $i=1, \ldots, n$, be $n \geq 2$ fuzzy variables, and let $S_{i}(\alpha)$ denote their one-dimensional $\alpha$-cuts, given by (4). We can consider three cases:

1. The fuzzy variables are said to be non-interactive if their $n$-dimensional joint $\alpha$-cut $S(\alpha)$, defined by (5), is given by the Cartesian product $S_{1}(\alpha) \times \ldots \times S_{n}(\alpha)$.

2. The fuzzy variables are said to be completely interactive if their $n$-dimensional joint $\alpha$-cut $S(\alpha)$ is the main space diagonal of the hyperrectangle $S_{1}(\alpha) \times \ldots \times S_{n}(\alpha)$.

3. The fuzzy variables are said to be partially interactive if they are neither non-interactive nor completely interactive.

In the present paper, as we will see in Sections 5 and 6, we need only non-interactive and completely interactive fuzzy variables. Hence, in what follows, we will not consider partially interactive fuzzy variables.

\subsection{Fuzzy functions}

A fuzzy function is a generalization of the concept of a classical function. A classical function is a mapping from its domain of definition into its range. Two important generalizations include: 1) a crisp map with fuzzy arguments; and 2) a fuzzy map with crisp arguments. Both cases generate a fuzzy output. In the present work, we only consider the first case and refer to a crisp map with fuzzy arguments as a fuzzy function.

The computation of fuzzy functions and operations on fuzzy functions can be carried out using the notion of $\alpha$-cuts as follows $[21,36]$. Let $\tilde{z}_{i}=\left\{\left(z_{i}, \mu_{i}\left(z_{i}\right)\right)\right\}$, with $i=1, \ldots, n$, be $n \geq 1$ completely interactive fuzzy variables with one-dimensional $\alpha$-cuts $S_{i}(\alpha)$ and an $n$-dimensional joint $\alpha$-cut $S(\alpha)$, which is the main space diagonal of the hyperrectangle $S_{1}(\alpha) \times \ldots \times S_{n}(\alpha)$. Set $\mathbf{z}=\left(z_{1}, \ldots, z_{n}\right)$, and let $g: Z \rightarrow W$ be a continuous mapping such that $\mathrm{w}=g(\mathbf{z})$. Then the $\alpha$-cut $S_{\mathrm{w}}(\alpha)$ corresponding to the membership function of the output fuzzy variable $\mathrm{w}$ in space $W$ is given by $S_{\mathrm{w}}(\alpha)=g(S(\alpha))$. This result decomposes fuzzy computations into several interval computations [62].

We now present a practical approach for fuzzy computations based on the above $\alpha$-cut representation. Our approach computes the output $\alpha$-cuts in three major steps, outlined in Algorithm 1 through a simple example.

Eventually, after computing the output $\alpha$-cuts for various $\alpha$ 's, e.g. $\alpha=0,0.1, \ldots, 1$, we can easily construct the membership functions of the output fuzzy variables. 
Algorithm 1 Fuzzy computations

Example. Compute the output $\alpha$-cuts of $\mathbf{w}_{ \pm}=g_{1}(\mathbf{z}) \pm g_{2}(\mathbf{z})$, where $g_{1}$ and $g_{2}$ are two continuous functions with $n$ fuzzy $\operatorname{arguments} \mathbf{z}=\left(z_{1}, \ldots, z_{n}\right)$, whose one-dimensional $\alpha$ cuts are $S_{1}(\alpha), \ldots, S_{n}(\alpha)$, with $\alpha \in[0,1]$.

1. Interaction. Find the joint $\alpha$-cut $S(\alpha)$ of the $n$ input fuzzy variables based on their interaction: $S(\alpha)$ is either the hyperrectangle $S_{1}(\alpha) \times \ldots S_{n}(\alpha)$ or its space diagonal, if the input fuzzy variables are either non-interactive or completely interactive, respectively.

2. Optimization. Solve the following optimization problems over the $n$-dimensional joint $\alpha$-cut $S(\alpha)$, which is the main space diagonal of the hyperrectangle $S_{1}(\alpha) \times \ldots \times S_{n}(\alpha)$ :

$$
g_{1}:=\min _{\mathbf{z} \in S(\alpha)} g_{1}(\mathbf{z}), \quad \overline{g_{1}}:=\max _{\mathbf{z} \in S(\alpha)} g_{1}(\mathbf{z}), \quad g_{\underline{2}}:=\min _{\mathbf{z} \in S(\alpha)} g_{2}(\mathbf{z}), \quad \overline{g_{2}}:=\max _{\mathbf{z} \in S(\alpha)} g_{2}(\mathbf{z}) .
$$

This can be done by employing a global optimization method [69]. Alternatively, we can employ numerical discretization. For instance, we uniformly discretize the intervals $S_{1}(\alpha), \ldots, S_{n}(\alpha)$ into $M$ discrete points $\left\{z_{1}^{(j)}\right\}_{j=1}^{M}, \ldots,\left\{z_{n}^{(j)}\right\}_{j=1}^{M}$. Then, if the $n$ input fuzzy variables are non-interactive, we evaluate the functions $g_{1}$ and $g_{2}$ on the full tensor product grid, i.e. the Cartesian product of the $n$ discretized intervals, containing $M^{n}$ grid points, and if the fuzzy variables are completely interactive, we evaluate the functions on $M$ grid points $\left\{\mathbf{z}^{(j)}\right\}_{j=1}^{M}$, where $\mathbf{z}^{(j)}=\left(z_{1}^{(j)}, \ldots, z_{n}^{(j)}\right)$. Finally, the global minima and maxima of the two functions over all grid points are calculated.

3. Best-case \& worst-case scenarios. Find the output $\alpha$-cuts based on the worst-case scenario (lower bound) and the best-case scenario (upper bound) among all possible outcomes:

$$
S_{\mathrm{w}_{+}}(\alpha)=\left[g_{1}+g_{2}, \overline{g_{1}}+\overline{g_{2}}\right], \quad S_{\mathrm{w}_{-}}(\alpha)=\left[g_{1}-\overline{g_{2}}, \overline{g_{1}}-g_{2}\right] .
$$

\subsection{Fuzzy random variables and fuzzy random functions}

A fuzzy random variable is a generalization of a random variable in probability theory. A random variable $y$ is a real-valued measurable function defined on a sample space $\Omega$. Every realization of a random variable $y(\omega)$, where $\omega \in \Omega$ is an outcome, is a real-valued number. To each measurable subset or event $A \subset \Omega$, a single precise probability measure $P(A)$ is assigned, satisfying Kolmogorov's axioms [43]. The probability can be described by, for instance, a cumulative distribution function (CDF). This concept can be generalized through combining probability theory and fuzzy set theory. A fuzzy-random variable w is a fuzzy-valued measurable function defined on the sample space $\Omega$. In other words, every realization of a fuzzy random variable $w(\omega)$ is a fuzzy variable, rather than a real-valued number. Correspondingly, a fuzzy probability measure and a fuzzy CDF will be assigned to a fuzzy-random variable; see Figure 8. We refer to [61] for more details on fuzzy randomness.

A fuzzy random function is a crisp map with a fuzzy vector and a random vector as arguments, generating an output fuzzy random variable. The computation of a fuzzy random function, outlined in Algorithm 2, amounts to computing the fuzzy CDF of its output fuzzy random variable; see Figures 11 and 15, which show the fuzzy CFDs of fuzzy random QoIs. 


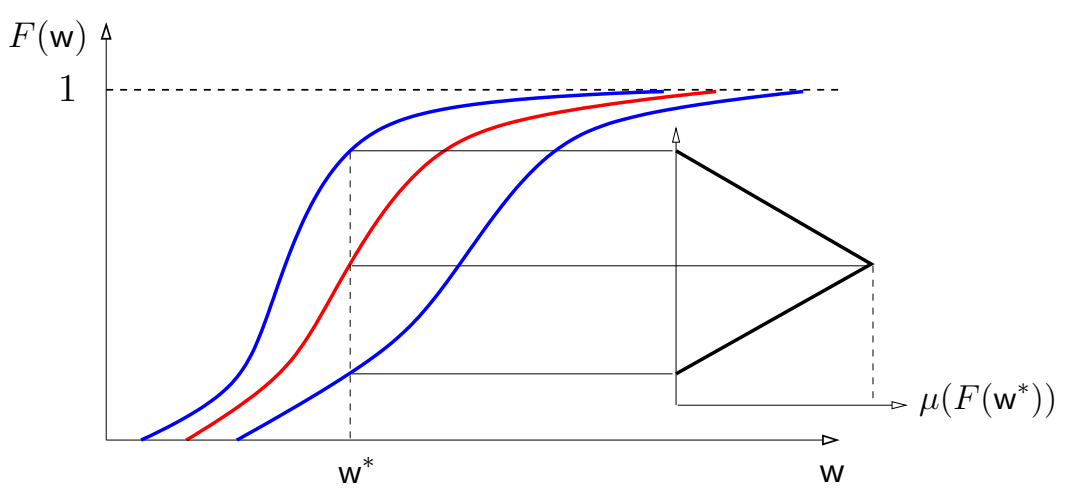

Figure 8: A schematic representation of the fuzzy CDF of a fuzzy random variable w. Each fixed realization $\mathrm{w}^{*}$ is a fuzzy variable. Only the 0 -cut (in blue) and 1-cut (in red) are shown.

\section{A hybrid fuzzy-stochastic model}

In this section, we construct and validate a new hybrid fuzzy-stochastic model for characterizing uncertainty in the input parameter $a(x)$ by combining stochastic fields and fuzzy variables. Motivated by the solution formula (3), we consider and study the reciprocal of the modulus of elasticity, i.e. $b(x)=a^{-1}(x)$. Our goal is to obtain an empirical model for the field $b(x)$ based on the real data collected in Section 3.1. We will motivate and explain the construction of the new model, consisting of a non-Gaussian random field whose first four statistical moments, i.e. mean, variance, skewness, and excess kurtosis are fuzzy variables.

Throughout this section, we consider a local small domain of length $L=10^{4}[\mu \mathrm{m}]$, and construct the model for this domain. In a similar way, models for domains of any size can be constructed. This particular choice will be used in Section 6 in the global-local approach.

\subsection{Fuzzy statistical moments}

In Section 3.2, we studied the first four statistical moments of $b(x)$ on a small domain of length $L=10^{4}[\mu \mathrm{m}]$. The data used in the statistical analysis were generated from the original set of data (50 samples of length $1700[\mu \mathrm{m}])$ by bootstrapping. We generated $M=100$ bootstrap samples of length $L=10^{4}[\mu \mathrm{m}]$, each at $N_{x}=10001$ discrete points $\left\{x_{j}\right\}_{j=1}^{N_{x}}$ on the interval $[0, \mathrm{~L}]$. We then used the bootstrap samples and obtained the histogram of the four sample moments; see Figure 4. We now want to model the four moments $\mu(x), \sigma(x), \gamma_{1}(x)$, and $\gamma_{2}(x)$, which vary in $x$, by four fuzzy variables $\tilde{z}_{1}, \tilde{z}_{2}, \tilde{z}_{3}, \tilde{z}_{4}$, respectively. This procedure is called the fuzzification of moments. We will fuzzify the moments using their histograms and construct four membership functions, denoted by $\mu_{1}\left(z_{1}\right), \ldots, \mu_{4}\left(z_{4}\right)$.

We first build up the histograms of the moments by dividing the range of the four sample moments (obtained by bootstrap samples) into 10 subintervals and determining the number of sample elements belonging to each subinterval; see Figures 4 and 9. As an initial draft for membership functions, we follow [61] and consider triangular membership functions consisting of two linear branches: a left and a right branch. The two linear branches are determined by the method of least squares as follows. The subinterval containing the largest number of sample elements is selected and called "mid-subinterval". The mid-subinterval and all subintervals to its left are used for computing the left branch of the membership function, 
Algorithm 2 Computation of fuzzy random functions

Example. Compute the fuzzy CDF of $\mathbf{w}=g(\mathbf{y}, \mathbf{z})$, where $g$ is a continuous fuzzy random function whose arguments an $N$-dimensional random vector $\mathbf{y}$ with known joint probability distribution and $n$ fuzzy variables $\mathbf{z}$ with known joint $\alpha$-cuts $S(\alpha)$.

1. Select $M_{s}$ independent samples of $N$ random variables $\left\{\mathbf{y}^{(m)}\right\}_{m=1}^{M_{s}}$.

2. Choose a fixed $\alpha \in[0,1]$ and uniformly discretize $S(\alpha)$ into $M_{\mathrm{f}}$ discrete points $\left\{\mathbf{z}^{(j)}\right\}_{j=1}^{M_{\mathrm{f}}}$. Note that if the fuzzy variables are non-interactive, the $n$-dimensional $S(\alpha)$ is a hyperrectangle, and if the fuzzy variables are completely interactive, the $n$-dimensional $S(\alpha)$ is embedded in a one-dimensional space.

3. For $m=1: M_{s}$

4. Calculate $\underline{g}^{(m)}:=\min _{j} g\left(\mathbf{y}^{(m)}, \mathbf{z}^{(j)}\right)$ and $\bar{g}^{(m)}:=\max _{j} g\left(\mathbf{y}^{(m)}, \mathbf{z}^{(j)}\right)$.

\section{End}

6. From the histograms of $\underline{g}$ and $\bar{g}$ and find their CDFs.

7. Go to step 2 and repeat for various $\alpha$ 's.

while the mid-subinterval and all subintervals to its right are used for computing the right branch. We collect two sets of left and right data points, whose $x$ - and $y$-coordinates are the mid points of selected subintervals and the corresponding number of sample elements, respectively. We then fit two lines to these two sets of data points by the least squares approach. The intersection of these two lines represent the mean value of the fuzzy variable. Note that the intersecting point may not lie in the mid-interval. The two neighboring zeros of these linear functions mark the interval bounds of the support. See Figure 9. It is to be noted that the above procedure is to generate an initial draft for membership functions. We may need to conduct a subsequent modification by imposing additional constraints and corrections. Finally, the membership functions are normalized so that the function value at the mean value point is one.

Furthermore, since the four statistical moments are obtained from the same set of data and are directly related to each other, i.e. higher moments are obtained from lower moments, it is reasonable to assume that the four fuzzy variables $\tilde{z}_{1}, \tilde{z}_{2}, \tilde{z}_{3}, \tilde{z}_{4}$ are completely interactive. This property will significantly simplify computations in the fuzzy space, because as we discussed in Section 4, we only need to consider the main space diagonal of the four-dimensional hyper rectangle, which reduces a four-dimensional fuzzy space to one dimension.

\subsection{A fuzzy-stationary stochastic field}

We are now ready to construct an empirical fuzzy-stochastic model for the parameter $b(x)$. We denote the empirical fuzzy-stochastic field by $\tilde{b}(x, \mathbf{y}, \mathbf{z})$, where $\mathbf{y}=\left(y_{1}, \ldots, y_{N}\right)$ is a random vector containing $N$ random variables, and $\mathbf{z}=\left(z_{1}, z_{2}, z_{3}, z_{4}\right)$ is the vector of four fuzzy variables, i.e. the four fuzzy moments obtained in Section 5.1.

We first note that by the construction, the fuzzy mean of the field is independent of $x$, i.e. $\mathbb{E}[\tilde{b}(x, ., \mathbf{z})]=z_{1}$. The other three higher moments are also fuzzy and independent of 

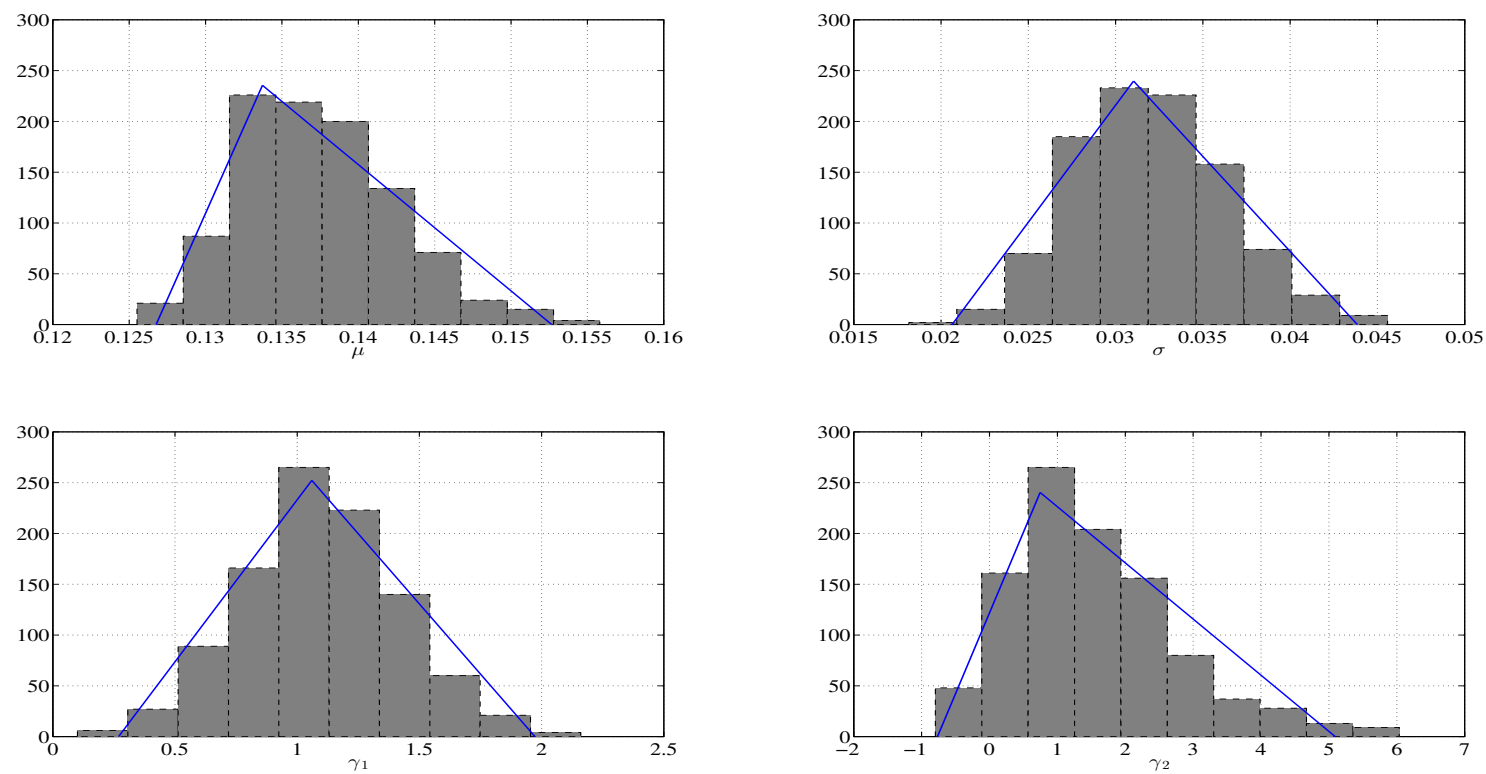

Figure 9: Histograms (gray bars) and non-normalized membership functions (blue solid lines) of sample mean (top left), sample standard deviation (top right), sample skewness (bottom left), and sample excess kurtosis (bottom right) of the field $b(x)=a^{-1}(x)$.

$x$. We may therefore call the field $\tilde{b}$ a fuzzy-stationary random field. Moreover, we notice that the field $\tilde{b}$ is not Gaussian, since for instance its skewness is not zero. Motivated by these observations and following the construction of stochastic translation processes [42], we propose a fuzzy-stationary non-Gaussian translation random field

$$
\tilde{b}(x, \mathbf{y}, \mathbf{z})=\Psi^{-1}(\mathbf{z}) \circ \Phi(G(x, \mathbf{y}))
$$

where $G(x, \mathbf{y})$ is a stationary Gaussian random field with mean zero and variance one, $\Phi$ is the standard Gaussian CDF, and $\Psi$ is a beta CDF with four parameters, chosen from the four fuzzy moments. For the stationary Gaussian field $G(x, \mathbf{y})$, we consider a squared exponential covariance function:

$$
C_{G}\left(x_{1}, x_{2}\right)=\exp \left(\frac{-\left|x_{1}-x_{2}\right|^{2}}{2 \ell^{2}}\right) .
$$

Motivated by the results of Section 3.3, we let the filed's spatial correlation length be $\ell=100$; see Figure 5. Since the covariance function (7) is deterministic, we employ the truncated Karhunen-Loéve expansion to represent the stationary Gaussian field:

$$
G(x, \mathbf{y}) \approx \sum_{n=1}^{N} \sqrt{\lambda_{n}} \phi_{n}(x) y_{n}
$$

where $\left\{\left(\lambda_{n}, \phi_{n}(x)\right)\right\}_{n=1}^{N}$ are the eigenpairs of the deterministic covariance function (7), and $\mathbf{y}=\left(y_{1}, \ldots, y_{N}\right) \in \mathbb{R}^{N}$ is a vector of $N$ uncorrelated normal random variables with zero mean and unit variance, i.e. $y_{n} \sim \mathcal{N}(0,1)$. The number of terms $N$ is chosen such that 
Algorithm 3 Construction of fuzzy-stochastic field based on the real data

0. Given a length $L$, obtain $M$ bootstrap samples from real data, as in Section 3.1.

1. Model first four moments by four fuzzy variables $\mathbf{z}=\left(z_{1}, z_{2}, z_{3}, z_{4}\right)$, as in Setion 5.1.

2. Compute a fuzzy beta distribution $\Psi(\mathbf{z})$ with four parameters given by four moments.

3. Generate a stationary Gaussian random field with covariance function (7) and correlation length $\ell=100$ by the truncated KL expansion (8).

4. Obtain the empirical fuzzy-stochastic field by (6).

a high percentage of the standard deviation is preserved. The recipe for constructing the fuzzy-stochastic field is outlined in Algorithm 3.

Model justification. We now perform a simple justification of the proposed fuzzy-stochastic model by showing that the CDFs of the true field $b(x)$ and the empirical fuzzy-stochastic field $\tilde{b}(x, \mathbf{y}, \mathbf{z})$ are in good agreement.

First, the CDFs of the true field $b(x)$ are obtained as follows. At each observation point $\left\{x_{j}\right\}_{j=1}^{N_{x}}$, we use $M$ realizations $\left\{b_{m}\left(x_{j}\right)\right\}_{m=1}^{M}$ and find the histogram of $b\left(x_{j}\right)$. We then normalize the histogram and obtain the corresponding CDF. See thin (turquoise) curves in Figure 10, which show $N_{x}$ CDFs of the true field $b(x)$ corresponding to $N_{x}$ observation points. Indeed, we obtain a probability box, or $p$-box, for the true field $b(x)$, which is another indication of the presence of imprecise uncertainty in the problem.

Next, we consider the empirical field $\tilde{b}(x, \mathbf{y}, \mathbf{z})$, constructed by Algorithm 3. In the particular case when $L=10^{4}[\mu \mathrm{m}]$ and $\ell=100[\mu \mathrm{m}]$, we need $N=42$ terms in the KL expansion to preserve $85 \%$ of the standard deviation. The random vector $\mathbf{y}$ therefore contains $N=42$ uncorrelated standard normal random variables. Different $\alpha$-cut intervals of the fuzzy vector $\mathbf{z}$, obtained in Section 5.1, give different $\alpha$-cuts of the fuzzy-stochastic field $\tilde{b}\left(x_{j}, \mathbf{y}, \mathbf{z}\right)$ at the $N_{x}$ observation point $\left\{x_{j}\right\}_{j=1}^{N_{x}}$. We employ Monte Carlo using $\hat{M}=10^{4}$ independent realizations of the normal random vector $\mathbf{y}$, and under the assumption that the fuzzy variables are completely interactive, we obtain the CDFs of the left and right limits of $\alpha$-cuts of the empirical field. Thick (brown) curves in Figure 10 show $N_{x}$ CDFs of the $\alpha$-cut for $\alpha=1$. We observe a good agreement between the CDFs of the true field and the CDFs of the constructed empirical field.

\subsection{Model Validation}

The fuzzy-stochastic model constructed in Section 5.2 needs to be validated based on the desired QoI. We note that a fuzzy-stochastic field on $x \in[0,1 \mathrm{~m}]$ turns the original problem (1) into a fuzzy-stochastic problem. This will turn the solution (3) into a fuzzy-stochastic field, explicitly given by $\tilde{u}(x, \mathbf{y}, \mathbf{z})=\int_{0}^{x} \tilde{b}(\xi, \mathbf{y}, \mathbf{z}) F(\xi) d \xi$.

In order to validate the proposed model, which is constructed on the small domain $[0, L]$ with $L=10^{4}[\mu \mathrm{m}]$, we first need to choose a QoI. For instance, we assume that the same problem as in (1) holds on the small domain $[0, L]$ with no external force term, i.e. $f \equiv 0$. 


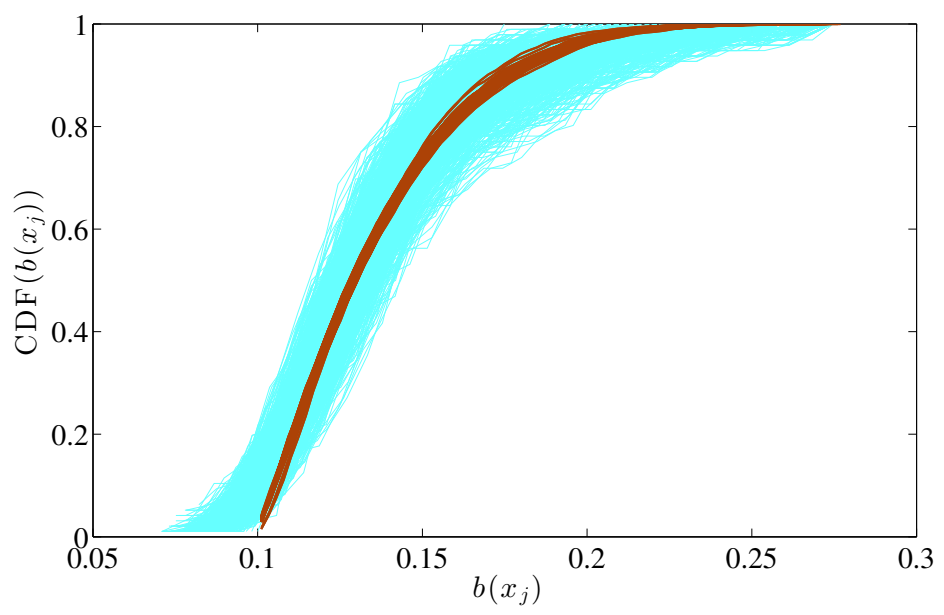

Figure 10: Thin (turquoise) curves are $N_{x}$ CDFs of the true field $b(x)$ which form a probability box. Thick (brown) curves are $N_{x}$ CDFs of the 1-cut of the empirical field $\tilde{b}(x, \mathbf{y}, \mathbf{z})$. Each CDF corresponds to a discrete point $\left\{x_{j}\right\}_{j=1}^{N_{x}}$ and is obtained from $M=100$ bootstrap samples.

We consider the solution at the mid-point $x=L / 2$ as the QoI, given by:

$$
\mathcal{Q}_{0}=\int_{0}^{L / 2} b(\xi) d \xi, \quad \tilde{\mathcal{Q}}_{0}(\mathbf{y}, \mathbf{z})=\int_{0}^{L / 2} \tilde{b}(\xi, \mathbf{y}, \mathbf{z}) d \xi .
$$

Here $\mathcal{Q}_{0}$ is the true QoI computed by the true field $b(x)$, and $\tilde{\mathcal{Q}}_{0}$ is the QoI computed by the proposed fuzzy-stochastic model $\tilde{b}(x, \mathbf{y}, \mathbf{z})$. We then proceed with a simple validation strategy $[13,14]$. We compare the true QoI directly obtained by the real data (i.e. $M=100$ bootstrap samples) and the left and right CDFs of the QoI obtained by the proposed model, as follows. First, we find a set of $N_{b}$ benchmark solutions, referred to as the truth. For this, we choose $N_{b}$ groups of samples, where each group consists of $\tilde{M}<M$ different, randomly selected samples out of $M$ bootstrap samples. For each group we then compute $\tilde{M}$ samples of the true quantity $\mathcal{Q}_{0}$ and then obtain its CDF. This gives us $N_{b}$ distributions of the true quantity $\mathcal{Q}_{0}$ in (9), which form a p-box and will be used as true validation data. Next, at different $\alpha$-cuts, we compute the CDFs of the left and right limits of the $\alpha$-cuts of the fuzzyrandom quantity $\tilde{\mathcal{Q}}_{0}$. This can for instance be done by employing a non-intrusive sampling technique, such as Monte Carlo, outlined in Algorithm 4.

Fuzzy integration. In step 4 of Algorith 4 , for a fixed realization of the random vector $\mathbf{y}^{(m)}$, the quantity $\tilde{\mathcal{Q}}_{0}$ in (9) is the integral of a fuzzy-valued function over a crisp interval. The proposed approach outlined in Algorithm 1 can easily be applied to compute the crisp integral of fuzzy functions. For instance, we can first approximate the crisp integral by a quadrature, such as trapezoidal rule:

$$
\tilde{\mathcal{Q}}_{0}\left(\mathbf{y}^{(m)}, \mathbf{z}\right)=\int_{0}^{L / 2} \tilde{b}\left(\xi, \mathbf{y}^{(m)}, \mathbf{z}\right) d \xi \approx \Delta x \sum_{j=1}^{N_{M}} \tilde{b}\left(x_{j}, \mathbf{y}^{(m)}, \mathbf{z}\right),
$$

where $\Delta x=L / 2\left(N_{M}-1\right)$. We next perform the addition of $N_{M}$ fuzzy-valued functions $\left\{\tilde{b}\left(x_{j}, \mathbf{y}^{(m)}, \mathbf{z}\right)\right\}_{j=1}^{N_{M}}$ of four completely interactive fuzzy variables, in the same way as the computation of the sum of two fuzzy functions presented in the example in Algorithm 1. 
Algorithm 4 Calculating fuzzy CDFs of the fuzzy-random QoI

1. Select $M_{s}$ independent samples of $N$ unit normal random variables $\left\{\mathbf{y}^{(m)}\right\}_{m=1}^{M_{s}}$.

2. $\forall \alpha$, uniformly discretize the $\alpha$-cut intervals of completely interactive fuzzy variables into $M_{\mathrm{f}}$ grid points $\left\{\mathbf{z}_{\alpha}^{(k)}\right\}_{k=1}^{M_{\mathrm{f}}}$.

3. For $m=1: M_{s}$

4. Calculate $\tilde{\mathcal{Q}}_{0 \alpha l}^{(m)}:=\min _{k} \tilde{\mathcal{Q}}_{0}\left(\mathbf{y}^{(m)}, \mathbf{z}_{\alpha}^{(k)}\right)$ and $\tilde{\mathcal{Q}}_{0 \alpha r}^{(m)}:=\max _{k} \tilde{\mathcal{Q}}_{0}\left(\mathbf{y}^{(m)}, \mathbf{z}_{\alpha}^{(k)}\right)$.

5. End

6. From the histograms of $\tilde{\mathcal{Q}}_{0 \alpha l}$ and $\tilde{\mathcal{Q}}_{0 \alpha r}$ and find their CDFs.

Figure 11, shows the p-box for the true quantity $\mathcal{Q}_{0}$ and the fuzzy CDFs of the approximate quantity $\tilde{\mathcal{Q}}_{0}$. As we see from the figure, the $\alpha$-cut of the approximate quantity, obtained by the proposed model can accurately capture the p-box of the true quantity. It is to be noted that the correlation length $\ell=100[\mu \mathrm{m}]$ is actually chosen so that the best fit is obtained.

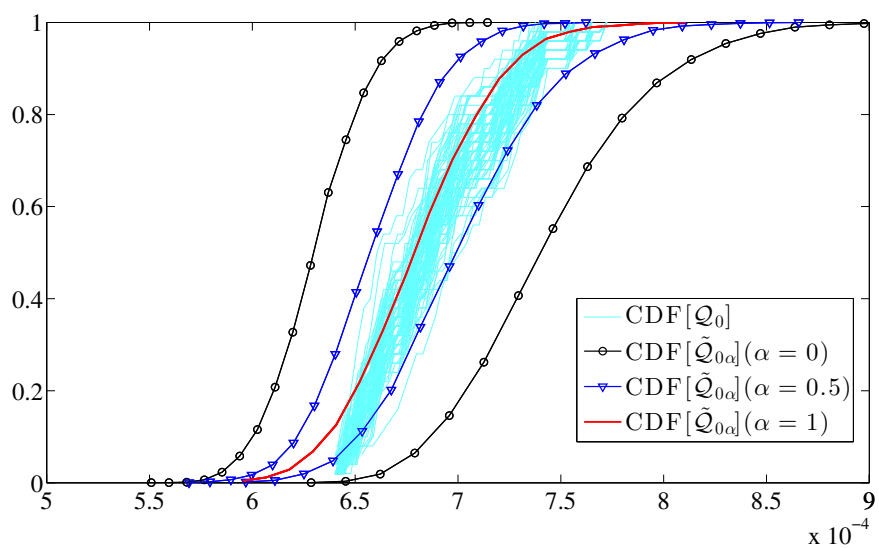

Figure 11: Probability box for the true QoI, directly obtained from the data, and fuzzy CDFs of the approximate QoI, computed by the proposed fuzzy-stochastic model.

\section{A global-local multiscale algorithm in fuzzy-stochastic framework}

In the mechanical design of fiber composites, we often need to determine the local features of the elastic field inside small parts of the domain. For instance, we may need to find the maximum stresses in small zones that are deemed vulnerable to failure. Such problems are amenable to global-local approaches, in which a global solution is used to construct a local solution that captures the microscale features of the true multiscale solution. In this section we develop a global-local technique, based on the proposed fuzzy-stochastic model, to compute the local features of the elastic field. We first start with a short description of the two tools that we need: RVE and homogenization.

\subsection{RVE and homogenization}

Consider a micro-structure where the size of the domain is sufficiently large relative to the typical inclusion size $h$. For instance, in the present work, the length of the composite 
plate $(1[\mathrm{~m}])$ is $10^{5}$ times larger than the typical size of fibers $(10[\mu \mathrm{m}])$. In general, an RVE is an element which is much smaller than the size of the overall domain and yet contains enough information on the micro-structure (here enough number of fibers) to describe the whole structure, see e. g. [51, 40]. Several definitions of an RVE have been proposed based on the above general definition:

- RVE is an element that: 1) is structurally entirely typical of the whole mixture on average; and 2) contains a sufficient number of inclusions for the apparent overall moduli to be effectively independent of the surface values of traction and displacement, as long as these values are macroscopically uniform [46].

- RVE must be chosen sufficiently large compared to the size of the microstructure for the approach to be valid, and it is the smallest material volume element of the composite for which the usual spatially constant overall macroscopic material properties are a sufficiently accurate model to represent the mean constitutive response [24].

- RVE is a model of the material to be used to determine the corresponding effective properties for the homogenized macroscopic model. An RVE should be large enough to contain sufficient information about the microstructure in order to be representative, however it should be much smaller than the macroscopic body. This is known as the Micro-Meso-Macro principle [45].

For micro-structures, an important issue is therefore to find the RVE size. In 1D problems, we need to find the RVE length, denoted by $L_{\mathrm{RVE}}$. By the above definition, the RVE length needs to be obtained from effective (or homogenized) properties. To find the RVE length, we proceed as follows; see also [51, 40]:

1. Generate a sequence of increasing element lengths $10 \mu \mathrm{m} \leq L_{1}<L_{2}<\ldots<L_{r} \ll 1 \mathrm{~m}$. For each $L_{i}$, with $i=1, \ldots, r$, we have $M$ samples available, obtained by bootstrapping.

2. For each fixed length $L_{i}$, we use homogenization (see below) with a homogenization length $H=L_{i}$ and obtain effective parameters for all $M$ samples, denoted by $\left\{b_{m}^{H}(x)\right\}_{m=1}^{M}$. We then compute the average of sample mean $\bar{\mu}\left[b^{H}\right]$ and the average of sample standard deviation $\bar{\sigma}\left[b^{H}\right]$ of the effective parameter. Note that the sample mean and standard deviation, obtained from $M$ samples, are functions of $x$ given on discrete points along $x$. Their average is taken over all discrete points along $x$.

3. Set up a criterion and a tolerance, such as:

$$
\varepsilon_{L}:=\frac{\bar{\sigma}\left[b^{H}\right]}{\bar{\mu}\left[b^{H}\right]} \leq \mathrm{TOL} .
$$

The smallest length $L$ that satisfies the criterion will be chosen as the RVE length $L_{\mathrm{RVE}}$. Note that the larger $L$, or equivalently the larger the homogenization length $H$, the less oscillatory the effective parameters $b_{m}^{H}(x)$ in $x$, and hence the smaller $\varepsilon_{L}$. 


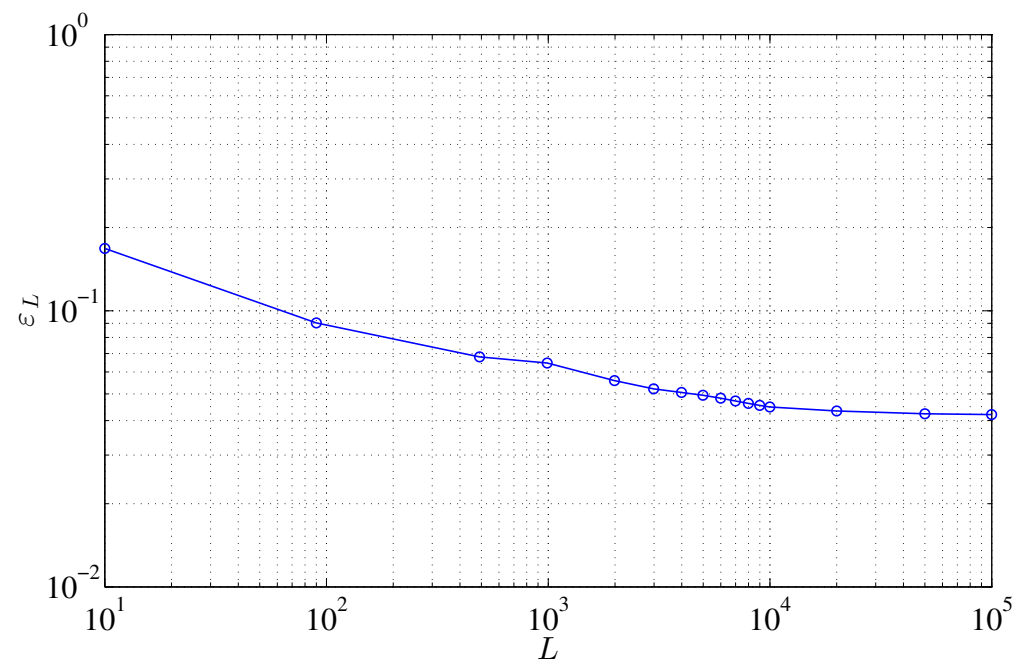

Figure 12: An RVE length $L_{\mathrm{RVE}}=10000[\mu \mathrm{m}]$ gives a relative error less than $5 \%$.

Figure 12 shows the relative error $\varepsilon_{L}$ versus $L$. We observe that the length $L=10^{4}[\mu \mathrm{m}]$ gives a relative error less than $5 \%$, and the decrease in the error for larger lengths is marginal. We therefore choose $L_{\mathrm{RVE}}=10^{4}[\mu \mathrm{m}]$.

Homogenization. A major step in finding the RVE length $L_{\mathrm{RVE}}$ described above is the computation of effective parameters on the RVE. The parameter $a(x)$ is highly oscillatory with variations of the size of fibers $(\sim h=10 \mu \mathrm{m})$, which are much smaller than the overall size of the composite $(\sim 1 \mathrm{~m})$. On a given domain of length $L>h$, we may therefore employ periodic homogenization, by periodically extending the highly oscillatory parameters $\left\{a_{m}(x)\right\}_{m=1}^{M}$ and obtain (effective) homogenized parameters $\left\{a_{m}^{H}(x)\right\}_{m=1}^{M}$, with $H$ being a homogenization length.

Consider a sample $m$ of length $L \ll 1 \mathrm{~m}$. For any given homogenization length $H$, we find the homogenized parameters $a_{m}^{H}(x)$ on the sample as follows. We first note that we have the highly oscillatory parameter $a_{m}(x)$ on the whole domain $x \in[0,1 \mathrm{~m}]$, given on square elements of size $h \times h$; see Figure 13 (middle).

The sample with length $L$ is first divided into $N_{x}$ square elements of size $h \times h$. In each element, we compute the homogenized parameter by taking the harmonic average over the surrounding $H / h$ elements of the sample with highly oscillatory parameters. This gives a homogenized parameter $a_{m}^{H}(x)$ on $N_{x}$ square elements centered at discrete points $\left\{x_{j}\right\}_{j=1}^{N_{x}}$. Figure 13 shows the procedure for two different homogenization lengths $H=30,50$. The smallest possible homogenization length in this setting is $H=h$, for which we have $a_{m}(x)=a_{m}^{H}(x)$, that corresponds to a full resolution of the micro scale. By repeating the same procedure for all samples $m=1, \ldots, M$, we collect $M$ realizations of the homogenized parameter $\left\{a_{m}^{H}(x)\right\}_{m=1}^{M}$ on a given length $L$. We will eventually use the homogenized parameters $\left\{b_{m}^{H}(x)\right\}_{m=1}^{M}$, where $b_{m}^{H}=1 / a_{m}^{H}$, to find the RVE length $L_{\mathrm{RVE}}$ and to construct the global model. 


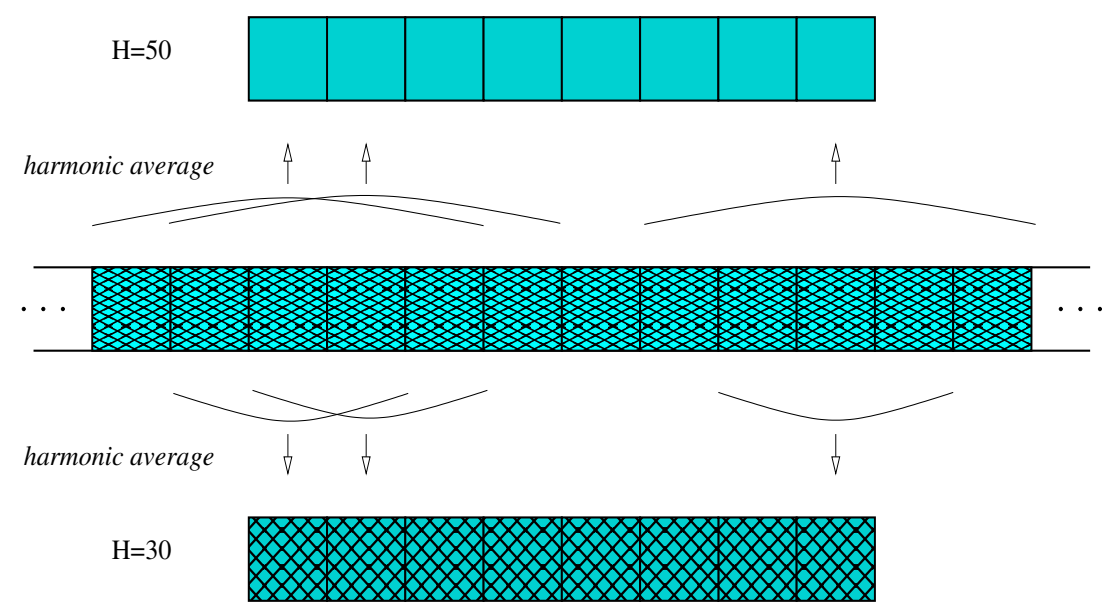

Figure 13: A schematic representation of obtaining 1D homogenized data from bootstrap data.

\subsection{Global-local algorithm}

We will follow a global-local approach and present an algorithm, outlined in Algorithm 5, consisting of two global and local parts. First, on the global domain $D=[0,1 \mathrm{~m}]$, we construct

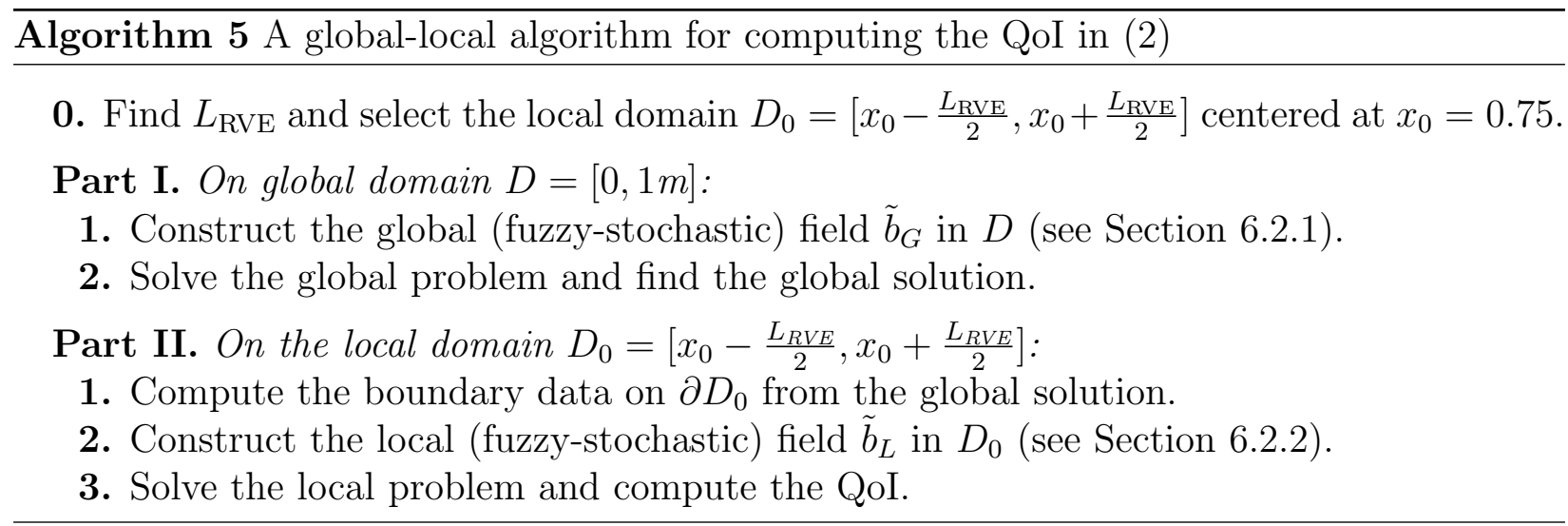

a global model parameter, say $\tilde{b}_{G}$, as described in Section 6.2.1, based on the homogenized data on the RVE. We then solve the global problem, which is the same as problem (1) with $a$ replaced by $\tilde{b}_{G}^{-1}$. The solution to the global problem is used to obtain boundary data for the local domain $D_{0} \subset D$, which is selected to be $D_{0}=\left[x_{0}-L_{\mathrm{RVE}} / 2, x_{0}+L_{\mathrm{RVE}} / 2\right]$. Inside the local domain $D_{0}$, we construct a local model parameter, say $\tilde{b}_{L}$, as described in Section 6.2.2. Finally, we solve the local problem, which consists of the same equation as (1a) with $f \equiv 0$ and Dirichlet boundary conditions with data obtained from the global solution, and compute the QoI.

\subsubsection{Global field}

The global field $\tilde{b}_{G}$ is constructed on the whole domain $D=[0,1 \mathrm{~m}]$, and based on the effective parameters on the RVE. The construction is similar to Algorithm 3 as follows:

1. Find $L_{\mathrm{RVE}}$ as described in Section 6.1. 
2. Take the homogenization length $H=L_{\mathrm{RVE}}$ and find effective parameters $\left\{b_{m}^{H}\left(x_{j}\right)\right\}_{j=1}^{N x}$ on the RVE for all samples $m=1, \ldots, M$, as described in Section 6.1. Note that we compute the effective parameters only on the RVE, not on the whole domain $[0,1 \mathrm{~m}]$.

3. Find the first four moments from the effective samples at all grid points $\left\{x_{j}\right\}_{j=1}^{N_{x}}$ and model them by fuzzy numbers. Similar to the four local fuzzy variables, the four global fuzzy variables are also completely interactive. Therefore, both global and local problems live in a one-dimensional fuzzy space. It is however important to note that since the global and local moments are obtained from two sets of different samples, i.e. homogenized and oscillatory samples, the two sets are non-interactive. Hence, the combined global-local problem lives in a two dimensional fuzzy space, which is the Cartesian product of two space diagonals.

4. Take the correlation length $\ell \sim 5 L_{\mathrm{RVE}}$ (see details below).

5. Construct the empirical global field $\tilde{b}_{G}$ as in Section 5. The global field will be a fuzzystationary non-Gaussian translation random field with a small number of KL terms, say $N_{I}$, and slowly varying in $x$. We write $\tilde{b}_{G}=\tilde{b}_{G}\left(x, \mathbf{y}_{I}, \mathbf{z}_{I}\right)$, where $\mathbf{y}_{I}$ is a vector of $N_{I}$ uncorrelated standard normal random variables, and $\mathbf{z}_{I}$ is a vector of four completely interactive fuzzy variables.

Correlation length for global field. Figure 14 shows the normalized correlation function $C_{m}\left(r_{n}\right)$ versus $r_{n}$, for two different homogenization lengths, $H=100,500$ and for several realizations $m=1, \ldots, M$. We observe that the correlation function decreases from 1 at $r=0$

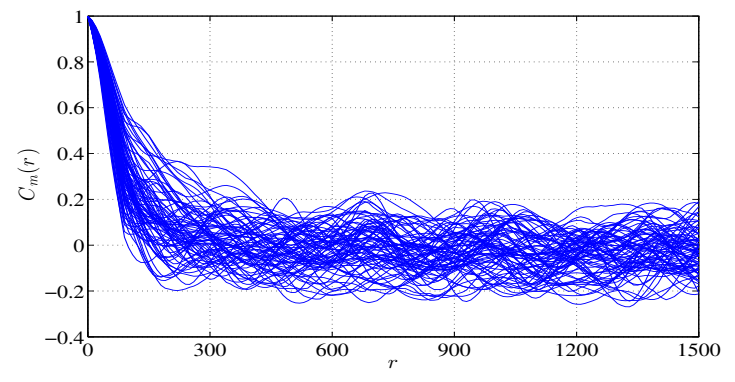

(a) $H=100$

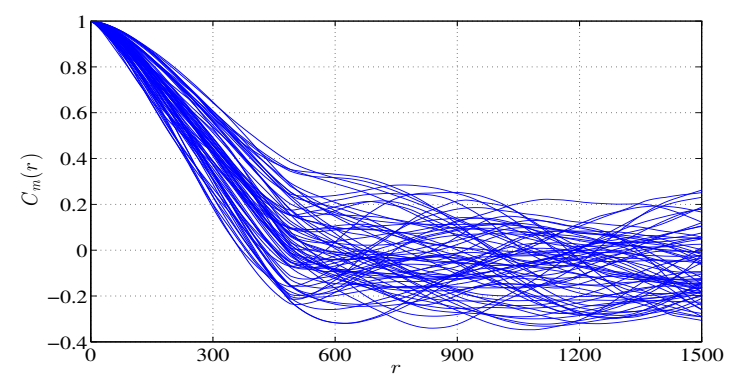

(b) $H=500$

Figure 14: Empirical correlation function $C_{m}\left(r_{n}\right)$ versus the distance $r_{n}$ for the homogenized parameter $b_{m}^{H}(x)$ with two different $H=100,500$ and for several realizations $m$.

by about $50 \%$ at $r=H$, and continues decreasing by $80 \%$ at larger distances. This suggests that the correlation length of the global field $b_{G}(x)$ is of the order of the homogenization length $(\sim 5 H)$. We will use this observation to construct the empirical global field. However, we note that the actual value of correlation length for the empirical field will be selected so that the best fit to the data is obtained. 


\subsubsection{Local field}

The local field $\tilde{b}_{L}$ is constructed on a local domain of size $L_{\mathrm{RVE}}$ and based on the highly oscillatory (non-homogenized) parameters on the local domain, in the same way as described in Algorithm 3. The local field will be a fuzzy-stochastic field $\tilde{b}_{L}=\tilde{b}_{L}\left(x, \mathbf{y}_{I I}, \mathbf{z}_{I I}\right)$, where $\mathbf{y}_{I I}$ is a vector of $N_{I I}$ uncorrelated standard normal random variables, and $\mathbf{z}_{I I}$ is a fuzzy vector of four completely interactive fuzzy variables.

\subsection{Validation}

Similar to Section 5.3, we need to validate the global-local model and approach. The validation needs to be done based on the true QoI given by (2) and (3):

$$
\mathcal{Q}=\int_{0}^{0.75} b(\xi) F(\xi) d \xi, \quad F(\xi)= \begin{cases}2 \xi & \xi \in[0,0.5), \\ 0 & \xi \in[0.5,1] .\end{cases}
$$

Here, $\mathcal{Q}$ is computed by the true field $b(x)$ on $[0,1 \mathrm{~m}]$. The approximate QoI, computed by the proposed model and the global-local approach, is obtained following Algorithm 5. The approximate QoI will be a fuzzy-stochastic field,

$$
\tilde{\mathcal{Q}}=\tilde{\mathcal{Q}}(\mathbf{y}, \mathbf{z}),
$$

where $\mathbf{y}=\left[\mathbf{y}_{I}, \mathbf{y}_{I I}\right]$ is a vector of $N=N_{I}+N_{I I}$ uncorrelated standard normal random variables, and $\mathbf{z}=\left[\mathbf{z}_{I}, \mathbf{z}_{I I}\right]$ is a vector of eight fuzzy variables, embedded in a two-dimensional fuzzy space. Note that $\mathbf{z}_{I}$ and $\mathbf{z}_{I I}$ are embedded in a one-dimensional space, because each of them contains four completely interactive fuzzy variables.

Similar to the procedure in Section 5.3, we can compare the p-box for the true quantity in (10) and the fuzzy CDFs of the approximate quantity in (11). As we see in Figure 15, the $\alpha$-cut of the approximate quantity, obtained by the proposed method can accurately capture the p-box of the true quantity.

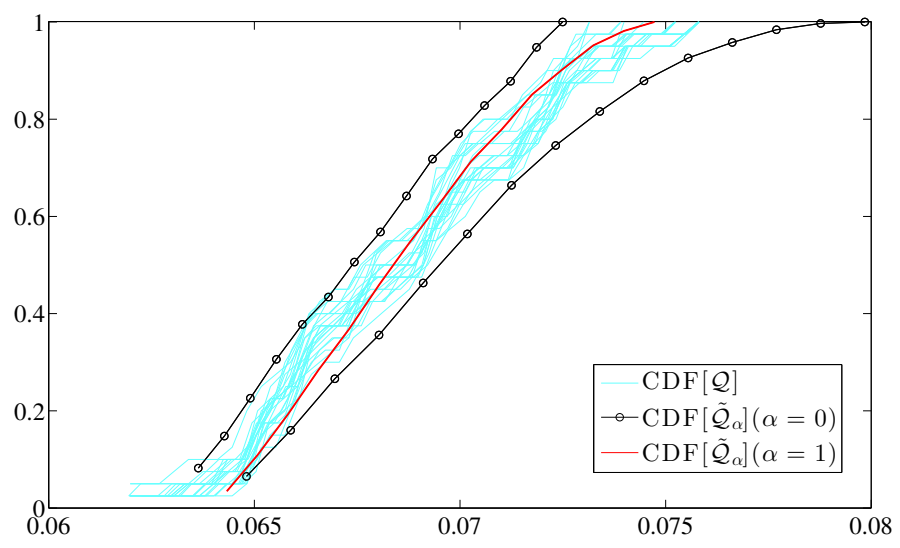

Figure 15: Probability box for the true QoI (thin turquoise curves) and CDF of the 1-cut of the approximate QoI (in black) computed by the fuzzy-stochastic global-local algorithm. 


\section{Conclusions}

First, we have shown that current stochastic multiscale models, such as stationary random fields, which are based on precise probability theory, are not capable of correctly characterizing uncertainty/variability in fiber composites. Next, we have motivated the applicability of mathematical models based on imprecise uncertainty theory and presented a novel hybrid fuzzy-stochastic model, which can more accurately describe uncertainty in fiber composites. The new model combines stochastic fields and fuzzy variables through a simple calibrationvalidation approach. Finally, we have constructed a global-local multiscale algorithm in a fuzzy-stochastic framework for efficiently computing output quantities of interest. The algorithm uses the concept of an RVE and homogenization and constructs a global solution to compute a local solution that captures the microscale features of the multiscale problem. The results are based on and backed by real experimental data.

For simplification and to motivate and establish the main concepts, we have considered a one-dimensional problem in the present work. Future directions include studying fiber composites in two and three dimensions and performing a rigorous error analysis for both the mathematical and computational models. Application to other engineering problems involving multiple scales and uncertainty is also a subject of our future work.

\section{Acknowledgements}

The first author is supported by NSF Grant DMS-1211014. The second author would like to recognize the support of the J. T. Oden Fellowship and the Institute for Computational Engineering and Sciences at the University of Texas at Austin.

\section{References}

[1] J. E. Aarnes and Y. Efendiev. Mixed multiscale finite element methods for stochastic porous media flows. SIAM J. Sci. Comput., 30:2319-2339, 2008.

[2] B. V. Asokan and N. Zabaras. A stochastic variational multiscale method for diffusion in heterogeneous random media. Journal of Computational Physics, 218:654-676, 2006.

[3] B. M. Ayyub and G. J. Klir. Uncertainty Modeling and Analysis in Engineering and the Sciences. Chapman and Hall, Boca Raton, 2006.

[4] I. Babuška. Solution of problem with interface and singularities. In C. deBoor, editor, Mathematical Aspects of Finite Elements in Partial Differential Equations, pages 213277. Academic Press, New York, 1974.

[5] I. Babuška. Homogenization and its applications, mathematical and computational problems. In B. Hubbard, editor, Numerical Solutions of Partial Differential EquationsIII, (SYNSPADE 1975, College Park MD, May 1975), pages 89-116, New York, 1976. Academic Press. 
[6] I. Babuška. Homogenization approach in engineering. In R. Glowinski and J. L. Lions, editors, Computing Methods in Applied Sciences and Engineering, Second International Symposium December 1975, volume 134 of Lecture Notes in Economics and Mathematical Systems, pages 137-153. Springer-Verlag, 1976.

[7] I. Babuška. Solution of interface problems by homogenization. I. SIAM J. Math. Anal., 7:603-634, 1976.

[8] I. Babuška. Solution of interface problems by homogenization. II. SIAM J. Math. Anal., 7:635-645, 1976.

[9] I. Babuška. Solution of interface problems by homogenization. III. SIAM J. Math. Anal., 8:923-937, 1977.

[10] I. Babuška, B. Andersson, P. J. Smith, and K. Levin. Damage analysis of fiber composites, Part I: statistical analysis on fiber scale. Comput. Methods Appl. Mech. Engrg., 172:27-77, 1999.

[11] I. Babuška and R. Lipton. $l^{2}$-global to local projection: an approach to multiscale analysis. Math. Mod. Methods Appl. Sci., 21:2211-2226, 2011.

[12] I. Babuška, M. Motamed, and R. Tempone. A stochastic multiscale method for the elastodynamic wave equations arising from fiber composites. Computer Methods in Applied Mechanics and Engineering, 276:190-211, 2014.

[13] I. Babuška, F. Nobile, and R. Tempone. Reliability of computational science. Numer. Methods Partial Differ. Eq., 23:753-784, 2007.

[14] I. Babuška, F. Nobile, and R. Tempone. A systematic approach to model validation based on Bayesian updates and prediction related rejection criteria. Comput. Methods Appl. Mech. Engrg., 197:2517-2539, 2008.

[15] I. Babuška and J. E. Osborn. Generalized finite element methods: Their performance and their relation to the mixed methods. SIAM J. Numer. Anal., 20:510-536, 1983.

[16] I. Babuška and R. S. Silva. Numerical treatment of engineering problems with uncertainties: the fuzzy set approach and its application to the heat exchange problem. Int. J. Numer. Meth. Engng., 87:115-148, 2011.

[17] A. Bensoussan, J.-L. Lions, and G. Papanicolau. Asymptotic Analysis for Periodic Structures. North-Holland, Amsterdam, 1978.

[18] X. Blanc and C. Le Bris. Improving on homogenized coefficients in the periodic and quasi-periodic settings. Networks and Heterogeneous Media, 5:1-29, 2010.

[19] A. Bourgeata and A. Piatnitski. Approximations of effective coefficients in stochastic homogenization. Annales de l'Institut Henri Poincare (B) Probability and Statistics, 40:153-165, 2004. 
[20] C. Le Bris, F. Legoll, and F. Thomines. Multiscale finite element approach for weakly random problems and related issues. ESAIM: Mathematical Modelling and Numerical Analysis, 48:815-858, 2014.

[21] C. Carlsson, R. Fullér, and P. Majlender. Additions of completely correlated fuzzy numbers. In Proceedings of IEEE International Conference on Fuzzy Systems, pages 535-539, Budapest, 2004.

[22] R. V. Demicco and G. J. Klir (Eds.). Fuzzy Logic in Geoplogy. Elsevier Academic Press, San Diego, 2004.

[23] M. Dorobantu and B. Engquist. Wavelet-based numerical homogenization. SIAM J. Numer. Anal., 35:540-559, 1998.

[24] W. J. Drugan and J. R. Willis. A micromechanics-based nonlocal constitutive equation and estimates of representative volume element size for elastic composites. J. Mech. Phys. Solids, 44:497-524, 1996.

[25] D. Dubois and H. Prade. Fuzzy Sets and Systems-Theory and Applications. Academic Press, Inc., San Diego, 1980.

[26] W. E. Principles of multiscale modeling. Cambridge University Press, Cambridge, 2011.

[27] W. E and B. Engquist. The heterogeneous multiscale methods. Commun. Math. Sci., 1:87-132, 2003.

[28] W. E, B. Engquist, X. Li, W. Ren, and E. Vanden-Eijnden. Heterogeneous multiscale methods: A review. Commun. Comput. Phys., 2:367-450, 2007.

[29] Y. Efendiev and T. Y. Hou. Multiscale finite element methods. Springer, 2009.

[30] B. Efron. Better bootstrap confidence intervals. Journal of the American Statistical Association, 82:171-185, 1987.

[31] B. Efron and R. Tibshirani. An Introduction to the Bootstrap. Chapman and Hall, London, 1993.

[32] B. Engquist and O. Runborg. Projection generated homogenization. In N. Antonić, C. J. van Duijn, W. Jäger, and A. Mikelić, editors, Multiscale Problems in Science and Technology, Proceedings of the Conference on Multiscale Problems in Science and Technology, Dubrovnik, Croatia, September 2000, pages 129-150. Springer, Berlin, 2002.

[33] B. Engquist and P. E. Souganidis. Asymptotic and numerical homogenization. Acta Numer., 17:147-190, 2008.

[34] F. Feyel and J. L. Chaboche. A multilevel finite element method (FE2) to describe the response of highly non-linear structures using generalized continua. Computer Methods in Applied Mechanics and Engineering, 192:3233-3244, 2003. 
[35] J. Fish, Q. Yu, and K. Shek. Computational damage mechanics for composite materials based on mathematical homogenization. Int. J. Numer. Methods Eng., 45:1657-1679, 1999.

[36] R. Fullér. On generalization of Nguy en's theorem: A short survey of recent developments. In J. Fodor and R. Fullér, editors, Advances in soft computing, intelligent robotics and control, volume 8 of Topics in Intelligent Engineering and Informatics, pages 183-190. Springer, 2014.

[37] B. Ganapathysubramanian and N. Zabaras. Modeling diffusion in random heterogeneous media: Data-driven models, stochastic collocation and the variational multiscale method. Journal of Computational Physics, 226:326-353, 2007.

[38] M. G. D. Geers, V. G. Kouznetsova, and W. A. M. Brekelmans. Multi-scale computational homogenization: Trends and challenges. Journal of Computational and Applied Mathematics, 234:2175-2182, 2010.

[39] S. Ghosh. Micromechanical analysis and multi-scale modeling using the Voronoi cell Finite Element Method. CRC Press, 2011.

[40] I. M. Gitman, H. Askes, and L. J. Sluys. Representative volume: Existence and size determination. Engineering Fracture Mechanics, 74:2518-2534, 2007.

[41] A. Gloria and F. Otto. An optimal variance estimate in stochastic homogenization of discrete elliptic equations. Ann. Probab., 39:779-856, 2011.

[42] M. Grigoriu. Simulation of stationary non-Gaussian translation processes. Journal of Engineering Mechanics, 124:121-126, 1998.

[43] M. Grigoriu. Stochastic Calculus, Applications in Sciences and Engineering. Birkhäuser, Boston, 2002.

[44] J. Guilleminot, C. Soize, and D. Kondo. Mesoscale probabilistic models for the elasticity tensor of fiber reinforced composites: Experimental identification and numerical aspects. Mechanics of Materials, 41:1309-1322, 2009.

[45] Z. Hashin. Analysis of composite materials - a survey. J. Appl. Mech., 50:481-505, 1983.

[46] R. Hill. Elastic properties of reinforced solids: some theoretical principles. J. Mech. Phys. Solids, 11:357-372, 1963.

[47] T. Y. Hou. Numerical approximations to multiscale solutions in partial differential equations. In J. F. Blowey, A. W. Craig, and T. Shardlow, editors, Frontiers in Numerical Analysis, Universitext, pages 241-301. Springer, 2003.

[48] T. J. R. Hughes, G. R. Feijoo, L. Mazzei, and J. B. Quincy. The variational multiscale method. A paradigm for computationnal mechanics. Comput. Methods Appl. Mech. Engrg., 166:3-24, 1998. 
[49] L. M. Kachanov. Time of the rupture process under creep conditions. Bull. SSR Acad. Sci., 8:26-31, 1958.

[50] L. M. Kachanov. Introduction to continuum damage mechanics. Martinus Nijhoff Publishers, Dordrecht, Netherlands, 1986.

[51] T. Kanit, S. Forest, I. Galliet, V. Mounoury, and D. Jeulin. Determination of the size of the representative voleme element for random composites: statistical and numerical approach. International Journal of Solids and Structures, 40:3647-3679, 2003.

[52] P. Kanouté, D. P. Boso, J. L. Chaboche, and B. A. Schrefler. Multiscale methods for composites: A review. Arch Comput Methods Eng, 16:31-75, 2009.

[53] I. G. Kevrekidis, C. W. Gear, J. M. Hyman, P. G. Kevrekidis, O. Runborg, and C. Theodoropoulos. Equation-free, coarse-grained multiscale computation: Enabling microscopic simulators to perform system-level analysis. Comm. Math. Sci., 1:715-762, 2003.

[54] P. Ladeveze, G. Lubineau, and D. Marsal. Towards a bridge between the micro- and mesomechanics of delamination for laminated composites. Composites Science and Technology, 66:698-712, 2006.

[55] P. Ladeveze, G. Lubineau, and D. Violeau. A computational damage micro model of laminated composites. International Journal of Fracture, 137:139-150, 2006.

[56] P. Ladeveze and A. Nouy. On a computational strategy with time and space homogenization for structural mechanics. Computer Methods in Applied Mechanics and Engineering, 192:3061-3087, 2003.

[57] W. K. Liu, D. Qian, S. Gonella, S. Li, W. Chen, and S. Chirputkar. Multiscale methods for mechanical science of complex materials: Bridging from quantum to stochastic multiresolution continuum. International Journal for Numerical Methods in Engineering, 83:1039-1080, 2010.

[58] Y. Liu, M. S. Greene, W. Chen, D. A. Dikin, and W. K. Liu. Computational microstructure characterization and reconstruction for stochastic multiscale material design. Computer-Aided Design, 45:65-76, 2013.

[59] X. Ma and N. Zabaras. A stochastic mixed finite element heterogeneous multiscale method for flow in porous media. Journal of Computational Physics, 230:4696-4722, 2011.

[60] A.M. Matache, I. Babuška, and C. Schwab. Generalized p-FEM in homogenization. Numerische Mathematik, 86:319-375, 2000.

[61] B. Möller and M. Beer. Fuzzy Randomness. Springer, New York, 2004.

[62] R. E. Moore, R. B. Kearfott, and M. J. Cloud. Introduction to Interval Analysis. SIAM Press, Philadelphia, Pennsylvania, 2009. 
[63] F. Murat and L. Tartar. H-convergence. In A. Cherkaev and R. Kohn, editors, Topics in the Mathematical Modelling of Composite Materials, volume 31 of Progress in Nonlinear Differential Equations and Their Applications, pages 21-43. Springer, 1997.

[64] J. T. Oden and K. S. Vemaganti. Estimation of local modeling error and goal-oriented adaptive modeling of heterogeneous materials. I: error estimates and adaptive algorithms. J. Comput. Phys., 164:22-47, 2000.

[65] M. Papadrakakis and G. Stefanou (Eds.). Multiscale Modeling and Uncertainty Quantification of Materials and Structures. Springer, Cambridge, 2014.

[66] K. K. Phoon, H. W. Huang, and S. T. Quek. Simulation of strongly non-Gaussian processes using Karhunen-Loeve expansion. Probabilistic Engineering Mechanics, 20:188$198,2005$.

[67] K. Rohwer. Predicting fiber composite damage and failure. Journal of Composite Materials, 49:2673-2683, 2015.

[68] T. J. Ross. Fuzzy Logic with Engineering Applications. Wiley, Singapore, 2010.

[69] K. Scheerlinck, H. Vernieuwe, and B. De Baets. Zadeh's extension principle for continuous functions of non-interactive variables: A parallel optimization approach. IEEE Transactions on Fuzzy Systems, 20:96-108, 2012.

[70] P. E. Souganidis. Stochastic homogenization of Hamilton-Jacobi equations and some applications. Asymptotic Analysis, 20:1-11, 1999.

[71] C. Soutis and P. W. R. Beaumont (Eds.). Multi-scale modelling of composite material systemms: The art of predictive damage modeling. Woodhead Publishing Limited, Cambridge, 2005.

[72] S. Spagnolo. Convergence in energy for elliptic operators. In B. Hubbard, editor, Numerical solution of partial differential equations III, Synspade 1975, pages 469-499. Academic Press, 1975.

[73] B. Tranquart, P. Ladeveze, E. Baranger, and A. Mouret. A computational approach for handling complex composite microstructures. Composite Structures, 94:2097-2109, 2012.

[74] K. S. Vemaganti and J. T. Oden. Estimation of local modeling error and goal-oriented adaptive modeling of heterogeneous materials. II: a computational environment for adaptive modeling of heterogeneous elastic solids. Comput. Methods Appl. Mech. Engrg., 190:6089-6124, 2001.

[75] X. Wan and G. E. Karniadakis. Solving elliptic problems with non-Gaussian spatiallydependent random coefficients. Computer Methods in Applied Mechanics and Engineering, 198:1985-1995, 2009. 
[76] X. F. Xu and L. Graham-Brady. A stochastic computational method for evaluation of global and local behavior of random elastic media. Computer Methods in Applied Mechanics and Engineering, 194:4362-4385, 2005.

[77] L. A. Zadeh. Fuzzy sets. Information and Control, 8:338-353, 1965. 